\title{
Loss of c-REL but not NF-kB2 prevents autoimmune disease driven by FasL mutation
}

\author{
LA O’Reilly ${ }^{\star, 1,2}$, P Hughes ${ }^{1,3}$, A Lin ${ }^{1}$, P Waring ${ }^{4}$, U Siebenlist ${ }^{5}$, R Jain ${ }^{1,2}$, DHD Gray ${ }^{1,2}$, S Gerondakis $^{6,7}$ and A Strasser, ${ }^{*, 1,2,7}$
}

FASL/FAS signaling imposes a critical barrier against autoimmune disease and lymphadenopathy. Mutant mice unable to produce membrane-bound FASL (FasL ${ }^{\Delta m / \Delta m}$ ), a prerequisite for FAS-induced apoptosis, develop lymphadenopathy and systemic autoimmune disease with immune complex-mediated glomerulonephritis. Prior to disease onset, Fas ${ }^{\Delta m / \Delta m}$ mice contain abnormally high numbers of leukocytes displaying activated and elevated NF-kB-regulated cytokine levels, indicating that NF- $\kappa$ B-dependent inflammation may be a key pathological driver in this multifaceted autoimmune disease. We tested this hypothesis by genetically impairing canonical or non-canonical NF- $k B$ signaling in Fas ${ }^{\Delta m / \Delta m}$ mice by deleting the $c-R e l$ or $N F-\kappa B 2$ genes, respectively. Although the loss of NF- $\kappa B 2$ reduced the levels of inflammatory cytokines and autoantibodies, the impact on animal survival was minor due to substantially accelerated and exacerbated lymphoproliferative disease. In contrast, a marked increase in lifespan resulting from the loss of c-REL coincided with a striking reduction in classical parameters of autoimmune pathology, including the levels of cytokines and antinuclear autoantibodies. Notably, the decrease in regulatory T-cell numbers associated with loss of $\mathrm{c}$-REL did not exacerbate autoimmunity in FasL ${ }^{\Delta m / \Delta m} \mathrm{c}$-rel ${ }^{\prime-}$ mice. These findings indicate that selective inhibition of C-REL may be an attractive strategy for the treatment of autoimmune pathologies driven by defects in FASL/FAS signaling that would be expected to circumvent many of the complications caused by pan-NF- $K B$ inhibition.

Cell Death and Differentiation (2015) 22, 767-778; doi:10.1038/cdd.2014.168; published online 31 October 2014

Apoptotic death of autoreactive lymphocytes constitutes a major mechanism of immunological tolerance. Defects in the deletion of autoreactive Tand B lymphocytes, either in primary lymphoid organs $^{1-3}$ or in the periphery, ${ }^{2}$ can lead to autoimmune disease. The 'death receptor' FAS (CD95, APO-1), and its ligand FASL (CD95L, APO-1L), has critical roles in the removal of autoreactive lymphocytes in the periphery, ${ }^{4}$ and in contraction of immune responses and lymphocyte homeostasis. ${ }^{5}$ Accordingly, loss-of-function mutations in these genes cause lymphadenopathy and systemic lupus erythematosus (SLE)-like multiorgan autoimmune disease in mice ${ }^{6}$ and humans. ${ }^{7}$

FAS, which is expressed on the surface of many cell types, allows FASL-mediated killing of pathogen-infected or damaged cells, ${ }^{4}$ whereas its ligand (FASL) is thought to be present only on activated $\mathrm{T}$ cells and NK cells. ${ }^{4}$ Upon deposition in the plasma membrane, membrane-bound FASL (mFASL) can also be processed and shed as secreted FASL (sFASL). ${ }^{4}$ The generation of mice that lack mFASL but express sFASL $\left(F a s L^{\Delta m / \Delta m}\right)$ demonstrated unambiguously that only the former is essential for FAS-mediated cell killing, activationinduced T-cell death (AICD) and lymphocyte homeostasis. ${ }^{8}$ Although FasL ${ }^{\Delta m / \Delta m}$ mice lack functional $\mathrm{mFASL}$, they can still produce sFASL, which can engage FAS, an interaction postulated to trigger non-apoptotic signals, ${ }^{4,9}$ which promote inflammation and cytokine production. ${ }^{8,10}$ Consistent with this hypothesis, FasL ${ }^{\Delta m / \Delta m}$ mice develop SLE-like kidney disease, autoimmune allergic dermatitis and histiocytic sarcoma with higher incidence and accelerated rate compared with Fas $L^{\text {gld } / g l d}$ mice, ${ }^{8}$ which carry a mutation that impairs the function of both mFASL and sFASL.

The onset of disease in FasL $L^{\Delta m / \Delta m}$ mice is preceded by an elevation in immune cells with activated nuclear factor- $\kappa \mathrm{B}$ $(\mathrm{NF}-\kappa \mathrm{B})$ that coincides with elevated serum levels of NF- $k \mathrm{~B}-$ regulated inflammatory cytokines. ${ }^{8}$ The NF- $\kappa$ B family of transcription factors, comprising homodimers and heterodimers of the five related proteins RELA, RELB, c-REL, $\mathrm{NF}-\kappa \mathrm{B} 1$ (p105/p50) and NF- $\kappa \mathrm{B} 2$ (p100/p52), regulates the development, survival and function of innate and adaptive immune cells. ${ }^{11,12}$ Different NF- $\kappa B$ dimers retained in a latent cytoplasmic state by various $I_{K} \mathrm{~B}$ inhibitory proteins are differentially activated by the canonical and non-canonical arms of the NF-KB pathway in response to distinct upstream signals (Supplementary Figure S1). ${ }^{13}$ Antigen receptors and inflammatory cytokines, such as TNF, IL- $1 \beta$ and IL-6, are among the many signals that activate the canonical NF- $k \mathrm{~B}$ transcription factors (dimers of c-REL or RELA and p50/NF- $K$ $\mathrm{B} 1^{11,12}$ ) through IKK $\beta$-dependent proteasome-mediated

\footnotetext{
${ }^{1}$ Molecular Genetics of Cancer Division, The Walter and Eliza Hall Institute of Medical Research, Parkville 3052, Victoria, Australia; ${ }^{2}$ Department of Medical Biology, The University of Melbourne, Parkville 3010, Victoria, Australia; ${ }^{3}$ Department of Nephrology, The Royal Melbourne Hospital, Parkville 3052 , Victoria, Australia; ${ }^{4}$ Department of Pathology, The University of Melbourne, Parkville 3010 Victoria, Australia; ${ }^{5}$ Laboratory of Molecular Immunology, National Institute of Allergy and Infectious Diseases, NIH, Bethesda, MD, USA and ${ }^{6}$ Australian Centre for Blood Diseases and Department of Clinical Hematology, Monash University Central Clinical School, Melbourne 3004, Victoria, Australia

*Corresponding author: LA O'Reilly or A Strasser, Molecular Genetics of Cancer Division, The Walter and Eliza Hall Institute of Medical Research, 1G Royal Parade, Parkville 3052, Victoria, Australia. Tel: +61 39345 2555, Fax: +61 39347 0852. E-mail: oreilly@ wehi.edu.au or strasser@wehi.edu.au

${ }^{7}$ These authors share senior authorship.

Abbreviations: ANA, antinuclear autoantibodies; GN, glomerulonephritis; sFASL, secreted FASL; mFASL, membrane-bound FASL; mTEC, medullary thymic epithelial cells; AIRE, autoimmune regulator

Received 04.5.14; revised 01.9.14; accepted 08.9.14; Edited by G Melino; published online 31.10.14
} 
degradation of $I_{K} B$ inhibitory proteins (Supplementary Figure S1). Conversely, certain TNF receptor family members (e.g., BAFF-R) trigger the non-canonical NF- $K B$ pathway through IKKa-induced proteolysis of p100/NF- $k$ B2 bound to RELB, thereby generating dimers of $p 52 / N F-k B 2$ and RelB (Supplementary Figure S1). ${ }^{11,12}$ Canonical and noncanonical NF- $\kappa$ B transcription factors generally control different sets of genes, ${ }^{14}$ although a subset of genes is co-regulated by the two arms of the NF- $\kappa$ B pathway.

Abnormally elevated NF-KB activity features in various human autoimmune diseases, including SLE and rheumatoid arthritis, ${ }^{12,15,16}$ where it is thought to drive pathology by regulating the proliferation, survival and effector activities of immune cells. ${ }^{16-19}$ The functions that NF- $k B$-regulated transcriptional programs exert in the immune system, combined with our findings that leukocytes in $\mathrm{FasL}^{\Delta m / \Delta m}$ mice have abnormally elevated NF- $\kappa \mathrm{B}$ activity before disease onset, led us to investigate whether NF- $\kappa \mathrm{B}$ signaling is critical for this disease. Due to the profound deleterious consequences associated with systemic NF- $\kappa \mathrm{B}$ inhibition, ${ }^{20}$ we focused on investigating the impact of selective loss of $c-R E L$ or NF- $k$ B2 on autoimmune pathology in FasL ${ }^{\Delta m / \Delta m}$ mice. These NF- $k B$ transcription factors were chosen because of their selective function in the canonical or non-canonical NF- $\kappa$ B pathways, and because they are mainly required for immune cell function and not for embryogenesis or hematopoietic cell development. Mice lacking c-REL, which is mainly expressed in hematopoietic cells, ${ }^{21}$ have no major abnormality but exhibit defective activation of B and T lymphocytes, macrophages and DC that collectively contribute to reduced antibody and cytokine production. ${ }^{12,22,23}$ In $n f k b 2^{-1-}$ mice, T-cell and myeloid cell development and functions are intact, but reduced mature B-cell numbers due to their impaired survival, combined with germinal center defects, result in reduced humoral immune responses. $^{24,25}$ Interestingly, with age, nfkb2 ${ }^{-1-}$ mice exhibit multiorgan leukocyte infiltrates and mild autoimmune disease. ${ }^{18,26}$ Our study demonstrates that loss of $c-R E L$ prevents autoantibody production in FasL ${ }^{\Delta m / \Delta m}$ mice and thereby substantially prolongs their lifespan. This suggests that selective targeting of $\mathrm{c}-\mathrm{REL}$ may represent a promising strategy for the treatment of systemic autoimmune diseases.

\section{Results}

Loss of c-REL extensively prolongs the lifespan of FasL $^{\Delta \mathrm{m} / \Delta \mathrm{m}}$ mice, whereas loss of NF- $\kappa$ B2 has only a modest impact. To investigate the roles of the canonical or non-canonical NF- $\kappa$ B signaling pathways in the lymphadenopathy and autoimmune disease caused by defects in FASL, we intercrossed FasL $L^{\Delta m / \Delta m}$ animals with $c-\mathrm{rel}^{\prime-}$ or $n f k b 2^{-1-}$ mice. Both the lifespan and pathology of the FasL ${ }^{\Delta m / \Delta m} c-r e l^{\prime-}$ and FasL ${ }^{\Delta m / \Delta m} n f k b 2^{-1-}$ compound mutants were compared with cohorts of wt, FasL ${ }^{\Delta m / \Delta m}, c-r e I^{\prime-}$ and $n f k b 2^{-1-}$ mice for a period of 2 years. Although the absence of NF- $k$ B2 or C-REL alone causes various immune defects, ${ }^{12,18}$ these animals had lifespans comparable to wt mice (wt versus nfkb2 ${ }^{\prime-}$ and wt versus $c-$ rel $^{\prime-}$ both $P=$ NS, Figure 1a). Loss of NF- $k \mathrm{~B} 2$ only modestly $(P=0.0344)$ prolonged the lifespan of $F a s L^{\Delta m / \Delta m}$ mice, whereas this was greatly extended by loss of $c-R E L(P<0.0001$, Figure $1 a)$.

The morbidity and premature death of $F_{a s}{ }^{\Delta m / \Delta m}$ mice are due to lymphadenopathy and SLE-like autoimmune glomerulonephritis (GN, Figure 1b). ${ }^{8}$ Histological examination (Figure 1c) and immunostaining for glomerular immune complex deposition indicated that the incidence of severe autoimmune kidney damage was significantly reduced in both FasL $^{\Delta m / \Delta m} \mathrm{C}-\mathrm{rel}^{\prime-}$ and FasL ${ }^{\Delta m / \Delta m} \mathrm{nfkb2}^{-1-}$ mice, and the damaging immunoglobulin deposition on the glomerular capillary loops (Figure 1d, arrows) in these animals was now mainly confined to mesangial cells (Figure 1d). Impairment of renal function (pathology grading 3-4) was modest and delayed in FasL $^{\Delta m / \Delta m} n f k b 2^{-1-}$ mice (FasL ${ }^{\Delta m / \Delta m} n f k b 2^{-1-}$ versus FasL ${ }^{\Delta m / \Delta m}$, $P<0.05)$. Loss of $\mathrm{c}-\mathrm{REL}$ had a more profound impact on GN-associated autoimmune pathology $(P=0.0002)$, manifest as substantial reductions in hypercellularity, capillary loop obliteration and fibrinoid necrosis (Figures $1 \mathrm{~b}$ and $\mathrm{c}$ ).

NF- $\kappa$ B2 and c-REL are required for inflammatory cytokine production in FasL ${ }^{\Delta \mathrm{m} / \Delta \mathrm{m}}$ mice. Before the onset of SLE-like autoimmune disease ( $\sim$ months), Fas ${ }^{\Delta m / \Delta m}$ mice harbor high levels of circulating inflammatory cytokines. ${ }^{8}$ We examined the levels of 31 cytokines and chemokines and found that TNF, IL-17A/F, KC (CXCL1) and MIP-1 $\beta$ levels were elevated in FasL $^{\Delta m / \Delta m}$ mice at 5 months of age (Figure 2a), whereas IFN $\beta$ remained largely undetectable by ELISA (Supplementary Figure S2), although type I interferons have been implicated previously in the GN in Fas ${ }^{I p r / p r}$ mutant mice. ${ }^{27} \mathrm{NF}-\mathrm{KB} 2$ deficiency blunted the excess production of most cytokines (except KC) in FasL ${ }^{\Delta m / \Delta m}$ mice (Figure 2a). As FasL ${ }^{\Delta m / \Delta m}$ mice aged, IL-1 $\beta$ and IL- 6 levels increased significantly, whereas in aged FasL ${ }^{\Delta m / \Delta m} n f k b 2^{-1-}$ mice (283-683 days) all cytokines tested, with the exception of IL-17A/F and $\mathrm{KC}$, remained at wt levels (Figure 2b). Remarkably, none of the cytokines or chemokines tested were elevated in FasL ${ }^{\Delta m / \Delta m} \mathrm{C}^{-r e l}{ }^{\prime-}$ mice, even when aged (Figures $2 a$ and $b$ ). This indicates that $c-R E L$ and, to a lesser extent, NF-B2 are required for accumulation of the cytokines and chemokines associated with autoimmune pathology in FasL ${ }^{\Delta m / \Delta m}$ mice.

NF- $\boldsymbol{K}$ B2 restrains lymphadenopathy in $\mathrm{FasL}^{\Delta \mathrm{m} / \Delta \mathrm{m}}$ mice. Defects in AICD of T cells are a major cause of lymphadenopathy in mice and humans deficient in FASL/FAS function and are associated with the accumulation of 'unusual' TCRa $\beta^{+}$ $\mathrm{CD}^{+} \mathrm{CD}^{-}{ }^{-} \mathrm{CD} 8^{-} \mathrm{B}^{-22}{ }^{+}$(hereafter called DN) T cells. ${ }^{6,7}$ FasL $^{\Delta m / \Delta m}$ mice display lymphadenopathy and splenomegaly commencing from $\sim 3$ months of age (Figures $3 a$ and $b$ ). Surprisingly, loss of NF- $\kappa$ B2 greatly accelerated lymphadenopathy in FasL ${ }^{\Delta m / \Delta m}$ mice (Figures $3 a$ and b) with a fourfold increase in DN T cells compared with Fas $L^{\Delta m / \Delta m}$ controls (Figure $3 c$ ). By 3 months, these DN T cells comprised the majority of leukocytes in the lymph nodes $(81.9 \pm 2.8 \%)$ and spleens of FasL ${ }^{\Delta m / \Delta m} n f k b 2^{-1-}$ mice $(76.7 \pm 1.1 \%$, Figure 3c), resulting in a $>10$-fold increase in total lymph node and splenic cellularity compared with Fas $L^{\Delta m / \Delta m}$ mice (Figure $3 e$ ). In contrast, loss of c-REL had no impact on the onset or magnitude of lymphadenopathy and DN T-cell numbers in FasL $^{\Delta m / \Delta m}$ mice (Figures $3 a-e$ ). To assess whether the enhanced lymphadenopathy of FasL ${ }^{\Delta m / \Delta m} n f k b 2^{-1-}$ mice was 


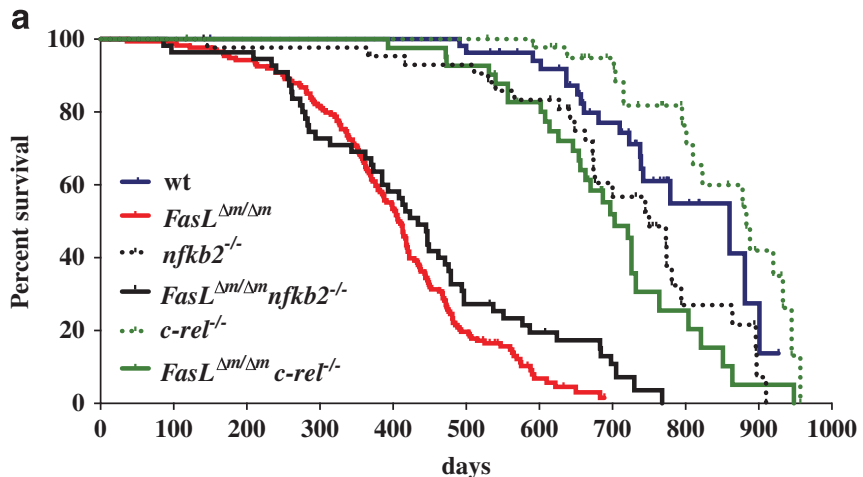

C
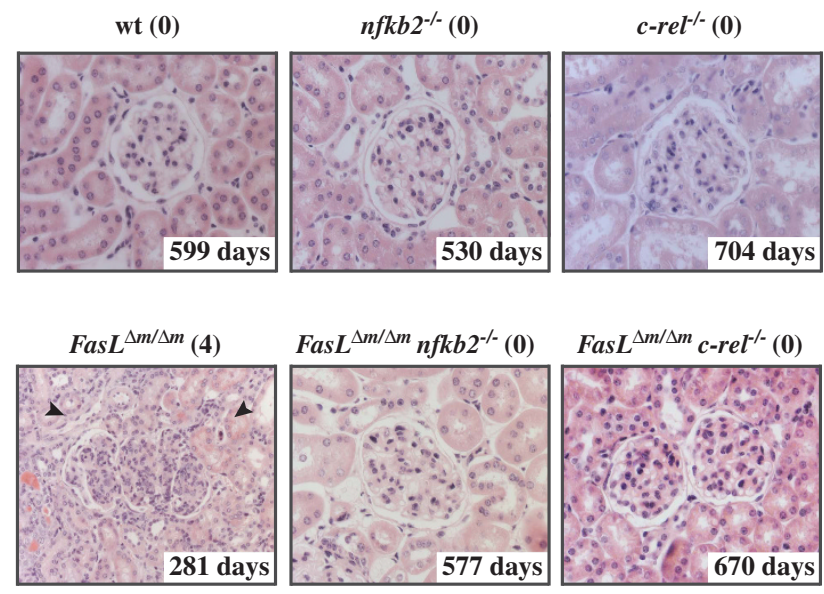

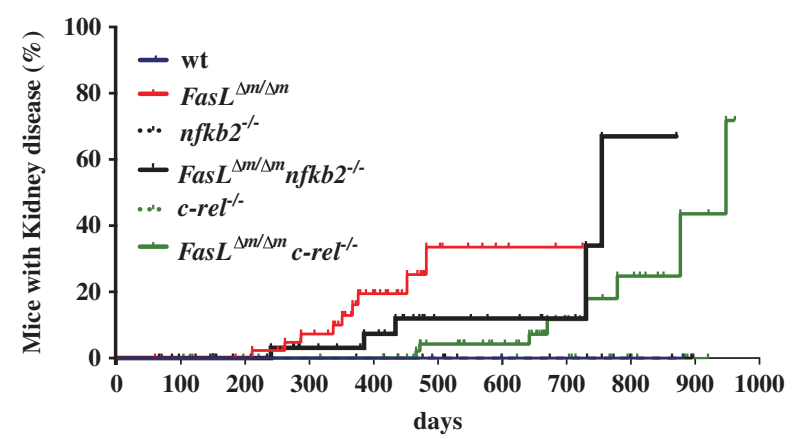

d

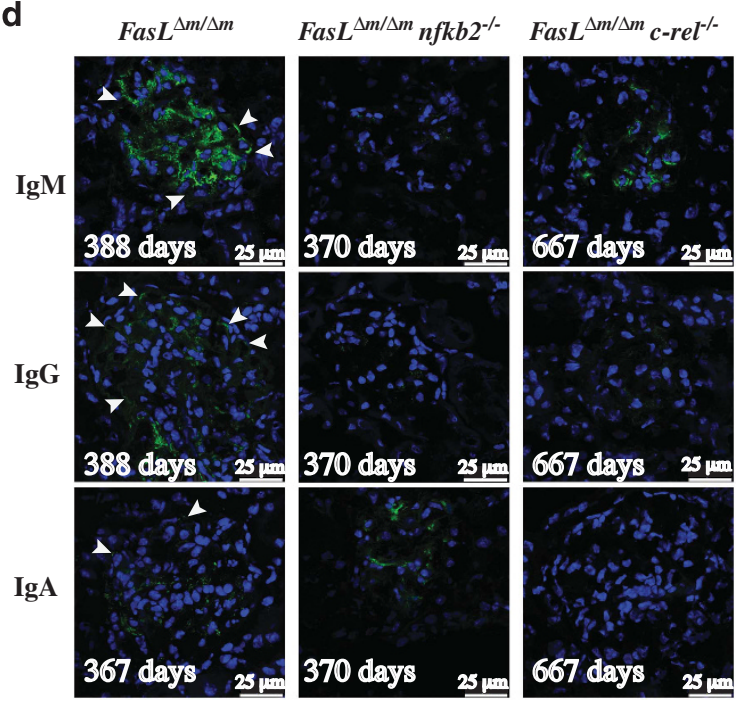

Figure 1 Loss of NF- $\kappa B 2$ or c-REL both prolonged the lifespan of FasL ${ }^{\Delta m / \Delta m}$ mutant mice and delayed or even prevented fatal SLE-like GN in these animals. (a) KaplanMeyer survival curves for wt $\left(n=54\right.$, mean survival 860 days, blue line), FasL ${ }^{\Delta m / \Delta m}\left(n=174\right.$, mean survival 407 days, red line), $n f k b 2^{-1-}(n=43$, mean survival 750 days, dotted black line), FasL ${ }^{\Delta m / \Delta m} n f k b 2^{-1-}\left(n=54\right.$, mean survival 434 days, solid black line), $c$-rel ${ }^{\prime-}\left(n=46\right.$, mean survival 883 days, dotted green line) and FasL ${ }^{\Delta m / \Delta m} c$-rel' ${ }^{\prime-}$ ( $n=40$, mean survival 703 days, solid green line) mice (wt versus FasL ${ }^{\Delta m / \Delta m}: P<0.0001 ; F^{2 a s}{ }^{\Delta m / \Delta m}$ versus FasL ${ }^{\Delta m / \Delta m} n f k b 2^{-1-}: P=0.0344 ;$ FasL ${ }^{\Delta m / \Delta m}$ versus FasL ${ }^{\Delta m / \Delta m}$

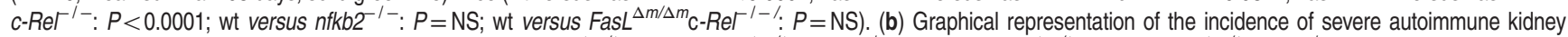
disease (GN score $\geq 3$ ) in mice of the indicated genotypes (FasL ${ }^{\Delta m / \Delta m}$ versus FasL ${ }^{\Delta m / \Delta m} n f k b 2^{-1}-: P<0.05$, FasL ${ }^{\Delta m / \Delta m}$ versus FasL ${ }^{\Delta m / \Delta m} \mathrm{C}$-rel ${ }^{\prime-}: P=0.0002$, lines colored

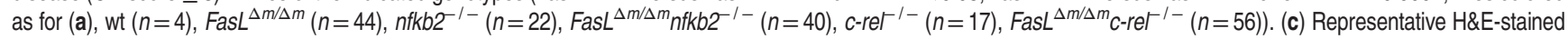
sections of the kidneys from mice of the indicated genotypes were examined for pathological changes, such as hypercellularity, cellular crescents, dilated tubules or sclerotic glomeruli. Arrows indicate fibrinoid necrosis. Magnification $\times 40$. (d) Representative confocal photomicrographs of frozen sections (eight kidneys per genotype analyzed) stained for the presence of IgA-, IgG- or IgM-containing immune complexes (green) in glomeruli. Nuclei are revealed by staining with DAPI (blue). Arrows indicate Ig deposition on the glomerular basement membrane (scale bars represent $25 \mu \mathrm{m}$ )

intrinsic to the hematopoietic system, bone marrow chimeras were generated. C57BL/6-Ly5.1 (wt) mice reconstituted with hematopoietic stem/progenitor cells (HSPCs) from Fas ${ }^{\Delta m / \Delta m}$ nfkb2 $2^{-1}$ mice exhibited enhanced lymphadenopathy and elevated numbers of DN T cells compared with mice reconstituted with FasL $^{\Delta m / \Delta m}$ HSPCs (Supplementary Figure S3a and c). This indicates that loss of NF- $k$ B2 in hematopoietic cells was sufficient to enhance the lymphadenopathy caused by defects in FASL.

Loss of c-REL or NF- $\kappa$ B2 reduces total antibody and autoantibody levels in FasL ${ }^{\Delta \mathrm{m} / \Delta \mathrm{m}}$ mice. FasL ${ }^{\Delta m / \Delta m}$ mice have elevated levels of antibodies of all $\mathrm{lg}$ isotypes (Figure $3 f$ and O'Reilly et al. ${ }^{8}$ ). In contrast, mice lacking NF-kB2 or c-REL have reduced levels of $\mathrm{Ig}$ isotypes requiring Ig heavy chain class switching (Figure $3 f$ and Franzoso et al. ${ }^{25}$, Köntgen et al. $^{28}$ ). With the exception of IgA in FasL ${ }^{\Delta m / \Delta m} n f k b 2^{-1-}$ mice, loss of either NF- $\kappa$ B2 or $c-R E L$ in FasL ${ }^{\Delta m / \Delta m}$ mice reduced all $\mathrm{Ig}$ isotype levels to those of wt mice (Figure 3f). High titers of antinuclear autoantibodies (ANA) producing a homogenous, nuclear staining pattern are a hallmark of SLE and have been observed in FasL ${ }^{\Delta m / \Delta m}$ mice (Figures $3 g-i$ and O'Reilly et al. ${ }^{8}$ ). Although the absence of NF-kB2 significantly reduced ANA levels in FasL ${ }^{\Delta m / \Delta m}$ mice $(P<0.005$; Figures $3 \mathrm{~g}-\mathrm{i})$, there was no difference in the $\mathrm{lg}$ isotypes among the ANA (Supplementary Figure S4). Remarkably, the sera of $\mathrm{FasL}^{\Delta \mathrm{m} / \Delta m_{\mathrm{C}-\mathrm{re}}{ }^{\prime-}}$ mice remained ANA negative (Figures $3 \mathrm{~g}-\mathrm{i}$ ).

To determine whether lymphocytic tissue infiltration, another feature of the autoimmune pathology in $\mathrm{FasL}^{\Delta m / \Delta m}$ mice, ${ }^{8}$ was associated with the production of organ-specific autoantibodies, we assessed the reactivity of sera from sick 

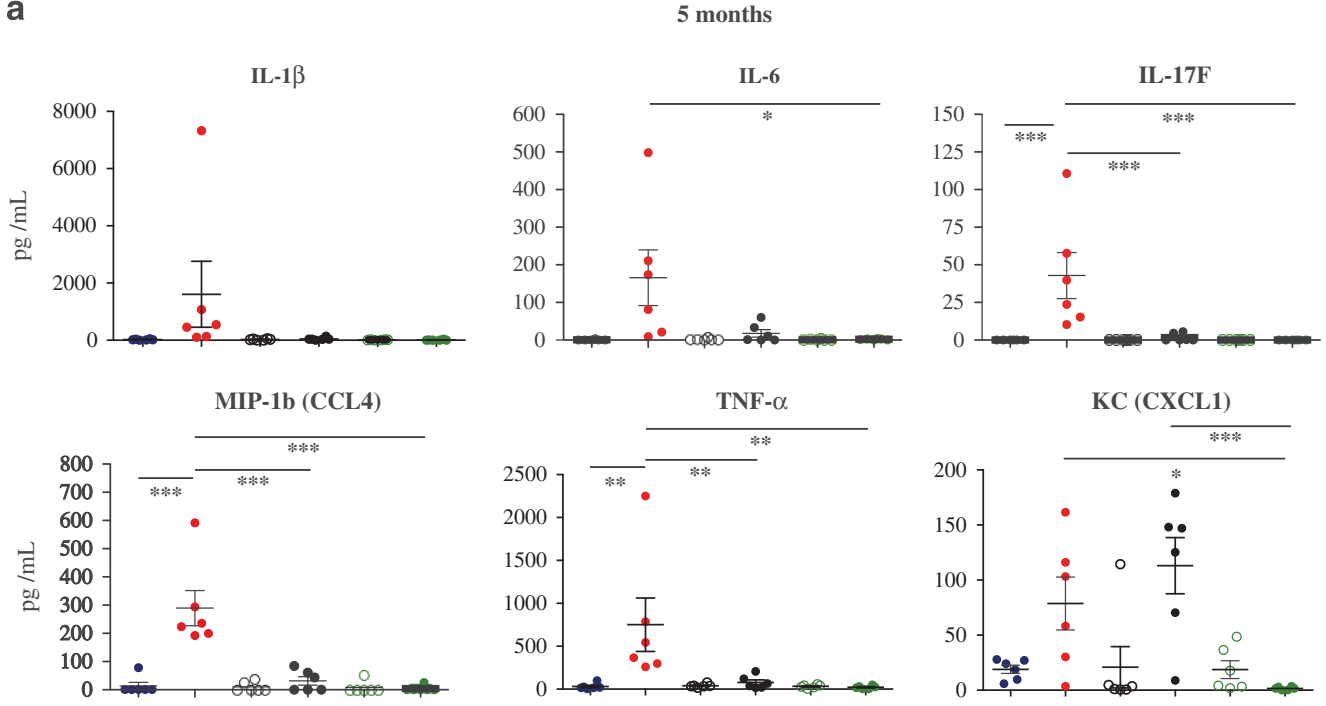

b

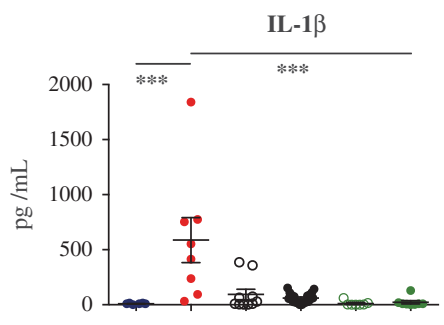

aged/sick
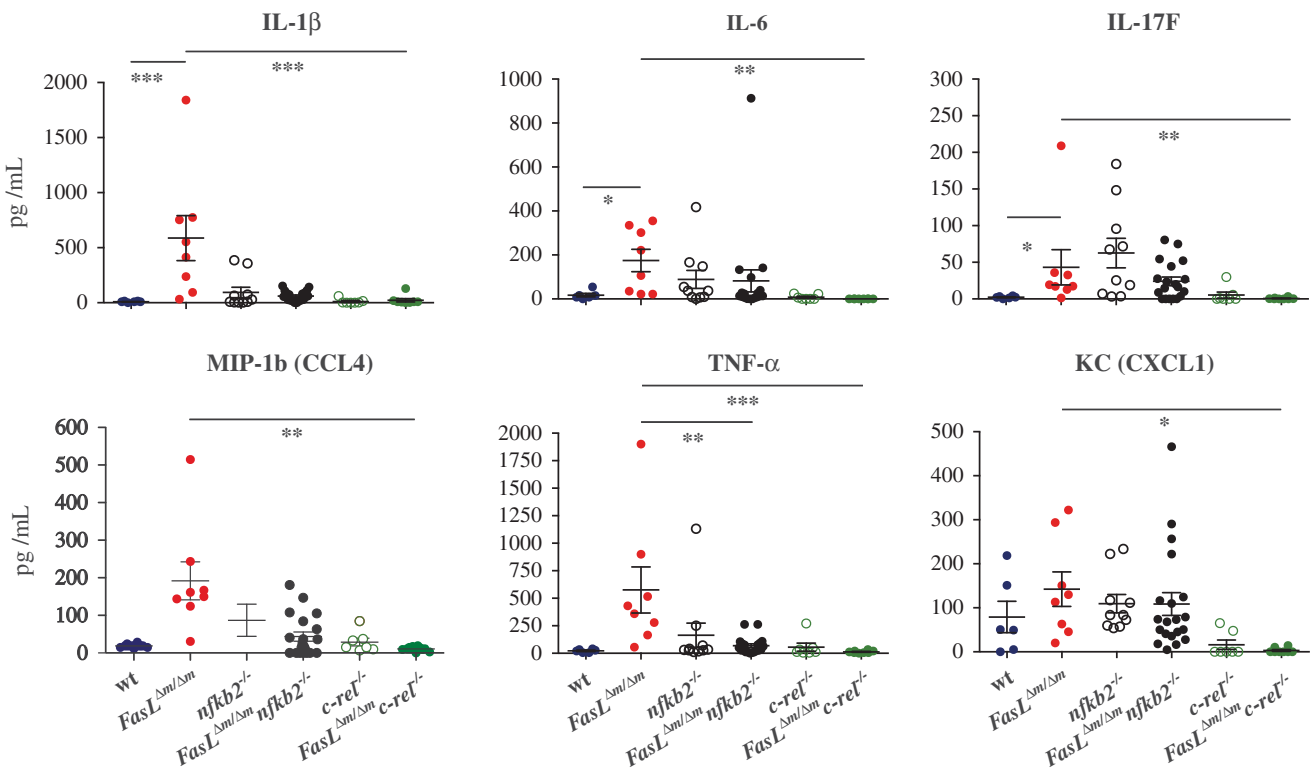

Figure 2 Impact of loss of c-REL or NF- $\kappa$ B2 on the levels of pro-inflammatory cytokines and chemokines in FasL ${ }^{\Delta m / \Delta m}$ mice. (a) The levels of 31 cytokines and chemokines (indicated in the Materials and Methods) were measured in the sera of mice of the indicated genotypes by the multiplex system at 5 months when animals still appeared healthy or (b) when they were terminally ill or aged. The levels of IL-1 $\beta$, IL-6, 1L-17F, CCL4, TNF- $\alpha$ and CXCL1 are shown. Each dot represents a single mouse; the bar indicates the average; the error bars represent S.E.M.

animals against a panel of tissues from rag-1 $1^{-1-}$ mice. Sick Fas $L^{\Delta m / \Delta m}$ mice harbored autoantibodies (IgM, IgA or $\operatorname{lgG}$ ) against lung epithelia, thyroid follicles, gastric parietal cells, submandibular gland and pancreatic acinar cells (Figure $3 \mathrm{f}$ ). No such autoantibodies were detected in the sera from similarly aged wt or $\mathrm{c}^{-\mathrm{rel}^{-1}}$ mice. Sera from $n f \mathrm{kb2}^{-1-}$ mice stained certain organs at a low level, and, although Fas $L^{\Delta m / \Delta m}$ $n f k b 2^{-1-}$ animals generally had lower autoantibody levels than FasL $\mathrm{Fm}^{\Delta m}$ mice, the persistent elevation of gastric autoantibodies may be due to the sustained serum IgA levels (Figure 3f). FasL ${ }^{\Delta m / \Delta m} C_{-}$rel $^{\prime-}$ mice had a substantial reduction in IgM, IgA or IgG autoantibodies, particularly those directed against the submandibular gland and thyroid, compared with $\mathrm{FasL}^{\Delta m / \Delta \mathrm{m}}$ animals (Supplementary Figure S5, Table 1).

The roles of NF-kB2 and c-REL in autoimmune pathology in Fas $L^{\Delta m / \Delta m}$ mice. To further understand how NF-KB2 and c-REL influence the early phase of autoimmune pathology in FasL $L^{\Delta m / \Delta m}$ mice, we examined the lungs, liver, salivary glands and pancreas at 5 months. Although lung disease was not apparent in $\mathrm{FasL}^{\Delta m / \Delta m}$ mice, severe peribronchial and perivascular lymphocytic infiltrates were prominent in $\mathrm{FasL}^{\Delta m / \Delta m} \mathrm{nfkb2}^{-1-}$ mice and constituted the main pathology (Figures $4 \mathrm{a}$ and $\mathrm{b}$ ). The infiltrates were predominantly composed of DN T cells with a polyclonal TCR $\beta$ variable 


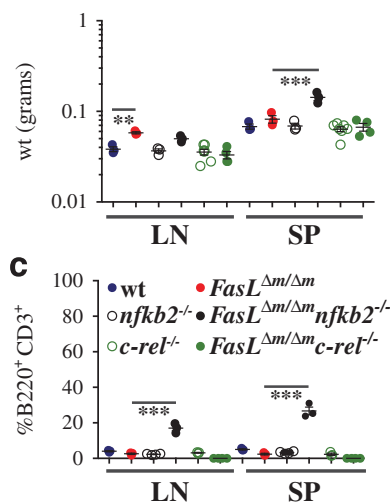

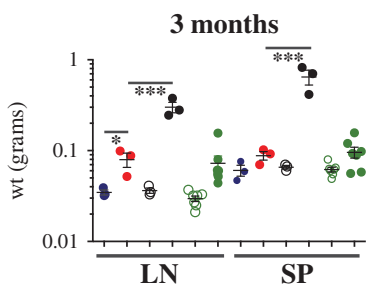
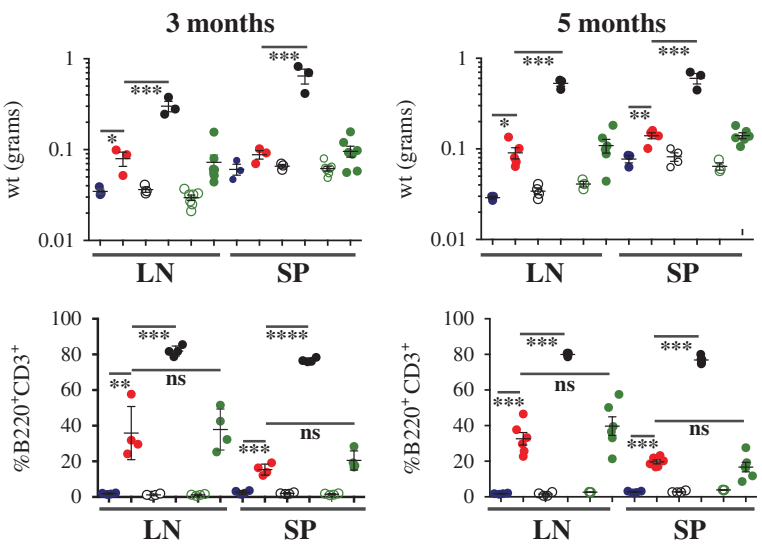
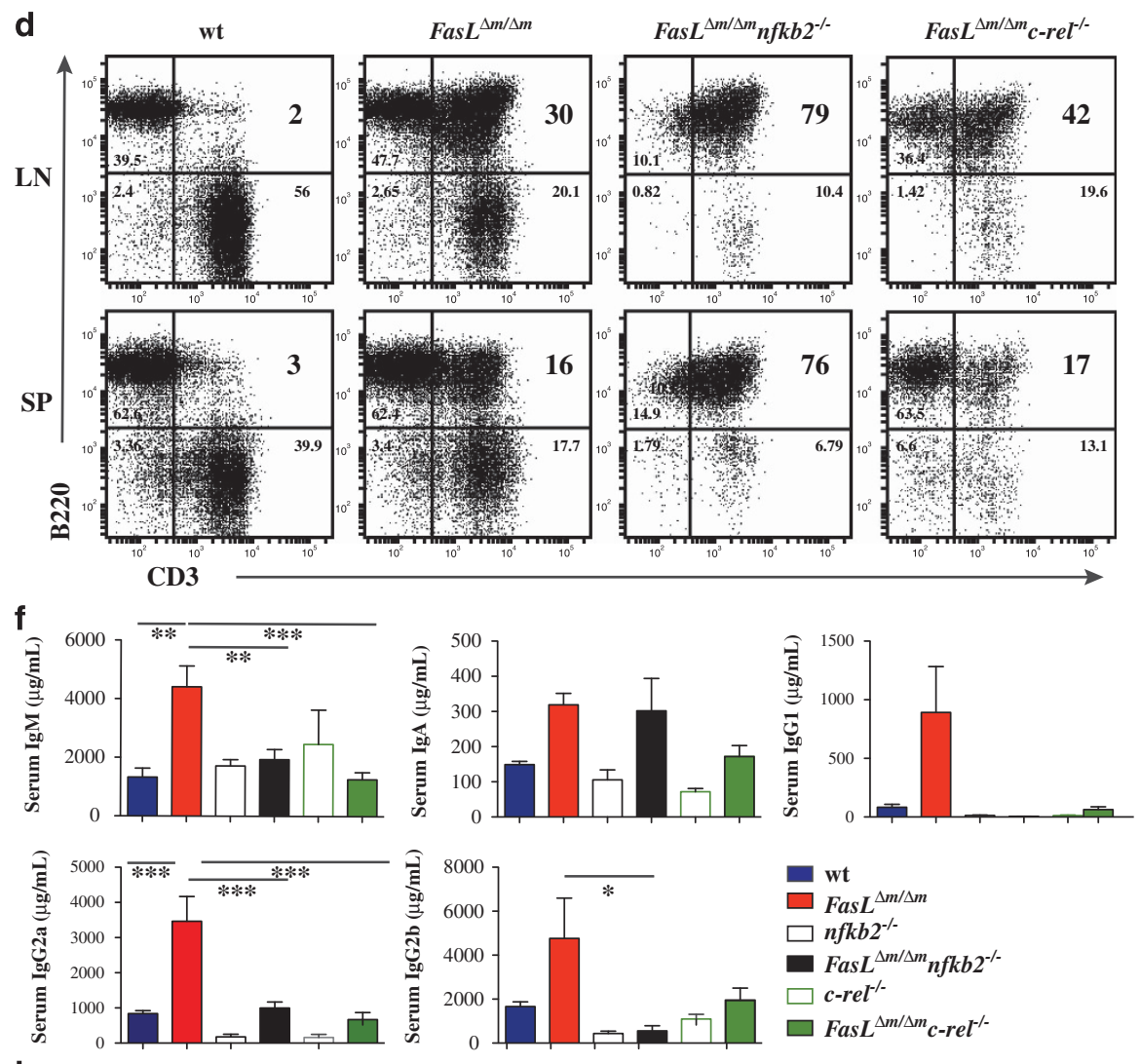

wt

$\square$ Fas $L^{\Delta m / \Delta m}$

$\square n f k b 2^{-/}$

Fas $L^{\Delta m / \Delta m} n f k b 2^{-/-}$

$\square \mathrm{c}-\mathrm{ret}^{-/-}$

$\square \mathrm{FasL}^{\Delta m / \Delta m} \mathrm{c}-\mathrm{rel} \mathrm{l}^{-/}$

i $\quad \operatorname{FasL}^{\Delta m / \Delta m}(+3)$
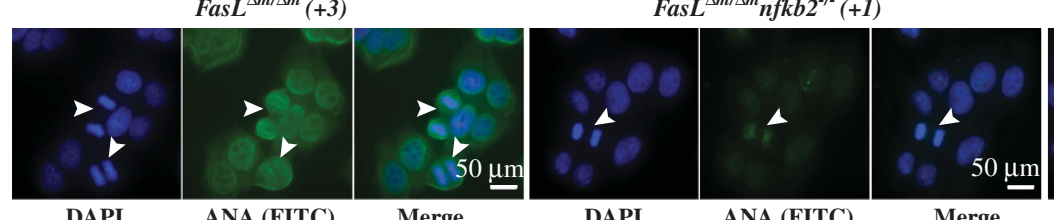

Merge

b

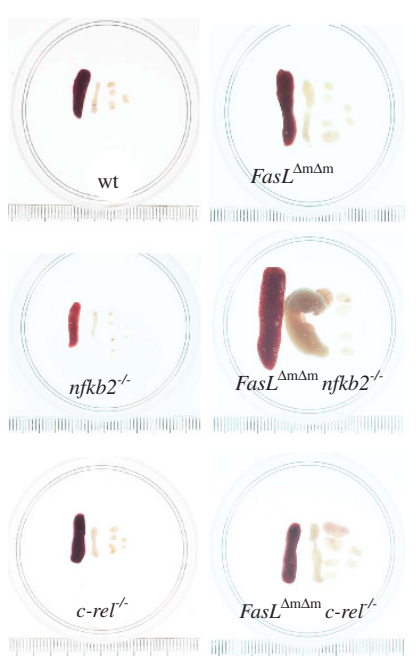

e

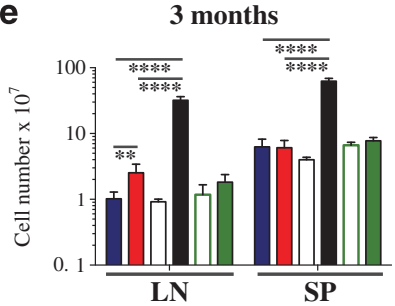

g

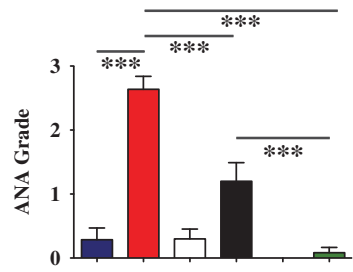

h

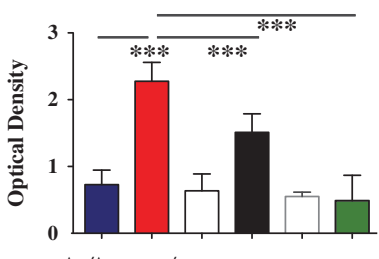

FasL $L^{\Delta m / \Delta m} c-r e l^{-/-}(0)$

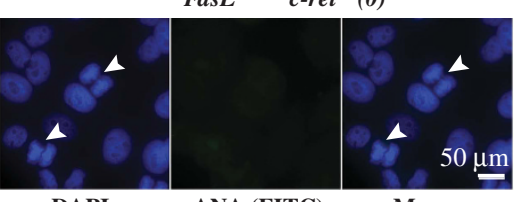

Figure 3 Loss of NF- $\kappa$ B2 accelerated and enhanced lymphadenopathy and splenomegaly in FasL ${ }^{\Delta m / \Delta m}$ mice and loss of NF- $\kappa$ B2 and c-REL both reduced hypergammaglobulinaemia and ANA in these animals. (a) Weights of the lymph nodes (axillary, brachial, inguinal and mesenteric) and spleens from mice of the indicated genotypes at the ages indicated. (b) Representative photomicrographs of the lymph nodes (axillary, brachial, inguinal and mesenteric) and spleen from mice of the indicated genotypes, all at $\sim 6$ months of age. Scale bar, each line represents $1 \mathrm{~mm}$. (c) The percentages of the 'unusual' DN T cells in the lymph nodes of mice of the indicated genotypes were measured by FACS analysis. In a and $\mathbf{c}$, each dot represents a single mouse and the figure legend in $\mathbf{c}$ also applies to $\mathbf{a}$. (d) Representative flow cytometric images of the DN T cells (numbers indicate percentage of cells in each quadrant). (e) Graph of total cell numbers for the indicated organs and the indicated genotypes of mice at 3 months of age ( $n=3-4$ mice/genotype). (f) The levels of antibodies of the different Ig isotypes in the sera of the mice of the indicated genotypes (age 5 months). Data represent mean \pm S.E.M. ( $n=6 /$ genotype). (g) ANA levels in the sera of mice of the indicated genotypes (5 months) were quantified by staining of human HEp-2 epithelial cells and scored according to a scale of fluorescence intensity on a scale of 0 (no fluorescence) to 3+ (maximal fluorescence). (h) Graphical representation of ANA levels in the sera of mice of the indicated genotypes quantified by ELISA ( $n=7-12$ mice/genotype). (i) Pictorial examples of ANA quantification in the sera of mice of the indicated genotypes; arrows indicate staining of nuclear components in mitotic figures. Immunofluorescence intensity score is indicated in brackets; ANA (green) and nuclei (blue) are revealed by staining with DAPI. Positive control sera were from Fas ${ }^{g l d g l d}$ mice. Values in graphs represent mean \pm S.E.M., ${ }^{\star} P<0.05,{ }^{\star *} P<0.001,{ }^{* \star \star} P<0.0001$ 
Table 1 Percentage of mice with organ-specific autoantibodies against the tissues indicated

\begin{tabular}{|c|c|c|c|c|c|c|c|c|c|}
\hline Strain & Salivary glands & Pancreas & Lacrimal glands & Lung & Liver & Thyroid & Stomach & $\mathbf{n}$ & Mean age \\
\hline wt (aged) & 0 & 0 & 20 & 0 & 0 & ND & 0 & 5 & $384 \pm 55$ \\
\hline FasL $^{\Delta m / \Delta m}$ (sick) & 75 & 63 & 50 & 100 & 100 & 100 & 100 & 8 & $369 \pm 97$ \\
\hline$n f k b 2^{-1-}$ (aged) & 25 & 25 & 13 & 38 & 25 & 0 & 25 & 8 & $392 \pm 24$ \\
\hline FasL $^{\Delta m / \Delta m}$ nfkb2 $2^{\prime-}$ (sick) & 25 & 38 & 63 & 75 & 13 & 0 & 100 & 8 & $291 \pm 79$ \\
\hline FasL $^{\Delta m / \Delta m} c-r e I^{\prime-}$ (aged) & 0 & 0 & 27 & 0 & 0 & 0 & 12 & 8 & $435 \pm 61$ \\
\hline
\end{tabular}

chain repertoire (Supplementary Figure S6), demonstrating their nonmalignant status.

Unlike sick FasL ${ }^{\Delta m / \Delta m} n f k b 2^{-1-}$ mice, which exhibited extensive lymphoid infiltrates and damage in the lungs, liver, pancreas and salivary glands, similarly aged sick Fas $L^{\Delta m / \Delta m}$ $(P<0.0001)$ and FasL ${ }^{\Delta m / \Delta m} C_{-r e l}{ }^{\prime-}$ mice $(P<0.0001)$ displayed milder pathology (Figure 4c). Aged or sick nfkb2-1mice only exhibited mild organ leukocyte infiltration (nfkb2 ${ }^{-1-}$ versus wt, $P<0.0001 ;$ nfkb2 ${ }^{-1-}$ versus $\mathrm{c}^{-\mathrm{rel}^{\prime-}}$ mice, $P<0.05)$. Many of the $\mathrm{CD} 4^{+} 8^{-}$and $\mathrm{CD} 4^{-} 8^{+} \mathrm{T}$ cells from the organs of aged or sick FasL ${ }^{\Delta m / \Delta m} n f k b 2^{-\prime-}$ mice were CD69+ (Figure 4d), indicating recent activation, possibly by selfantigens. Sick Fas ${ }^{\Delta m / \Delta m} n f k b 2^{-1-}$ mice (but none of the animals of the other genotypes) also suffered from multiorgan necrotizing vasculitis, an inflammatory disease characterized by damaged vessel walls, involving the kidney and occasionally other organs (Figure 4e,Supplementary Table S1).

$F_{a s L}{ }^{\Delta m / \Delta m}$ mice develop autoimmune dermatitis $(32 \%)$, which presents as reddening, thickening and scabbing of the ears and neck (Figure $4 \mathrm{~h}$ and O'Reilly et al. ${ }^{8}$ ). Interestingly, FasL $^{\Delta m / \Delta m}$ fkb2 $^{-/-}$mice did not develop autoimmune dermatitis, whereas $\mathrm{FasL}^{\Delta \mathrm{m} / \Delta m} \mathrm{C}_{-\mathrm{rel}} \mathrm{I}^{\prime-}$ mice showed a similar incidence of this disease (39\%), albeit with delayed onset compared with the FasL ${ }^{\Delta m / \Delta m}$ animals $(660 \pm 134$ days versus $435 \pm 86$ days) (Figures $4 f$ and h). Dermatitis in Fas $L^{\Delta m / \Delta m}$ and FasL ${ }^{\Delta m / \Delta m} \mathrm{C}$-rel $I^{\prime-}$ mice was associated with elevated serum IgE levels (Figure $4 \mathrm{~g}$ and O'Reilly et al. ${ }^{8}$ ), a feature shared with humans suffering from this autoimmune pathology. ${ }^{29}$ These findings show that loss of c-REL merely delayed autoimmune dermatitis in FasL ${ }^{\Delta m / \Delta m}$ mice, whereas loss of NF- $k$ B2 abrogated this pathology.

Regulatory T cells (Tregs) have a key role in the suppression of autoimmunity and modulation of the intensity and quality of immune responses to foreign antigens. ${ }^{30}$ Of relevance to this study, reductions in Treg numbers or impaired Treg function have been implicated in murine and human autoimmune dermatitis. $^{31,32}$ To ascertain whether this was the case in Fas $L^{\Delta m / \Delta m}$ mice and whether this was influenced by the absence of NF- $k$ B2 or c-REL, Tregs $\left(\mathrm{CD} 4^{+} \mathrm{CD} 25^{+} \mathrm{Foxp}^{+}\right)$ were enumerated in lymphoid tissues. Treg numbers in FasL $L^{\Delta m / \Delta m}$ and $n f k b 2^{-1-26}$ mice were normal (Figures 5a and b) and even elevated in the FasL ${ }^{\Delta m / \Delta m} n f k b 2^{-1-}$ mice (Figure 5b). However, consistent with a previous report ${ }^{33}$ showing that loss of c-REL resulted in a substantial reduction in Tregs, both the percentages and absolute numbers of Tregs in FasL ${ }^{\Delta m / \Delta m} \mathrm{C}_{\text {rel }}{ }^{\prime-}$ mice were markedly reduced compared with FasL ${ }^{\Delta m / \Delta m}$ and wt animals (Figures $5 a$ and b). These results, combined with the impact that NF-KB2 or c-REL loss had on the phenotype in Fas $L^{\Delta m / \Delta m}$ mice, indicate that while Treg defects are not likely to be critical for the development of autoimmune pathology in these animals reduction in Treg numbers may explain in part the dermatitis seen in the Fas $L^{\Delta m / \Delta m}$

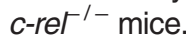

NF-KB2 but not mFASL or C-REL is required for the development of AIRE+ mTECs. The epithelial components of the thymic stroma have critical roles in promoting central tolerance to tissue-specific antigens. ${ }^{34} \mathrm{~A}$ reduction in the number or function of medullary thymic epithelial cells (mTECs) is linked to T-cell-dependent inflammatory diseases triggered by autoreactive $T$ cells that escape negative selection. ${ }^{34,35}$ Both the canonical and non-canonical NF-kB pathways have been implicated in tolerogenic processes mediated by the thymic stroma, ${ }^{36}$ with the non-canonical pathway reported to be important for the differentiation and/or survival of mTECs. ${ }^{34}$ NF-kB2 deficiency was associated with a loss of $C D 80^{+}$mTEC, but not the reduction of mTECs that express the autoimmune regulator (AIRE).$^{18}$ We conducted a detailed analysis of the thymic microenvironment to examine whether mTEC defects might be responsible for the organspecific autoimmunity observed in Fas ${ }^{\Delta m / \Delta m}$ mice or for the alterations in the $F a s L^{\Delta m / \Delta m}$ disease profile exerted by loss of NF-KB2 or c-REL (summarized in Table 2).

Thymi from 6- or 20-week-old FasL ${ }^{\Delta m / \Delta m}$ mice stained for mTEC markers (keratin-5 (K5), UEA-1 and AIRE) displayed a medullary architecture comparable to that of age-matched wt mice, although the levels of UEA-1 may be lower (Figures 6a and $b$ ). The expression of MTEC markers in the thymi of $c$-rel' ${ }^{\prime-}$ and $\mathrm{FasL}^{\Delta m / \Delta m} \mathrm{C}_{-\mathrm{rel}}{ }^{\prime-}$ mice was also normal (Figures $6 a$ and b). In contrast, thymi from $n f k b 2^{-1-}$ and $F a s L^{\Delta m / \Delta m}$

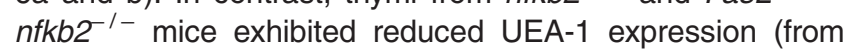
6 weeks) and an abnormal distribution of $\mathrm{K}^{+} \mathrm{mTEC}$ (from 20 weeks, Figures $6 a$ and b). Flow cytometric analysis indicated that the total numbers of epithelial cells were similar across all genotypes (Figure 6c) and the total numbers of cortical TEC (CTEC) and mTEC subsets expressing high or low levels of MHCII (mTEC hi and mTEC ${ }^{\text {low }}$ ) were comparable between wt, FasL ${ }^{\Delta m / \Delta m}, c-r e I^{\prime-}$ and FasL ${ }^{\Delta m / \Delta m} \mathrm{C}_{\text {-rel }}{ }^{\prime-}$ mice (Figure 6d). However, the numbers of mTEChi cells were substantially diminished in thymi of $n f k b 2^{-1-}$ and $F_{a s L^{\Delta m / \Delta m}}$ $n f k b 2^{-1-}$ mice (Figure 6e), reflected by an almost complete absence of AIRE $^{+}$TECs ( $10 \%$ of wt numbers). Interestingly, Fas $^{\Delta m / \Delta m}$ mice also displayed elevated CD4 CD8 ${ }^{+}$thymocyte numbers (Figure 6f). In summary, although FasL ${ }^{\Delta m / \Delta m}$ mice display many features of autoimmune disease, they have a normal TEC composition, indicating that MFASL has no major role in TEC development and survival. Loss of AIRE expressing mTECs, however, correlates with impaired negative selection and multiorgan autoimmunity ${ }^{34}$ and this may provide an explanation for the mild age-related autoimmunity 
a

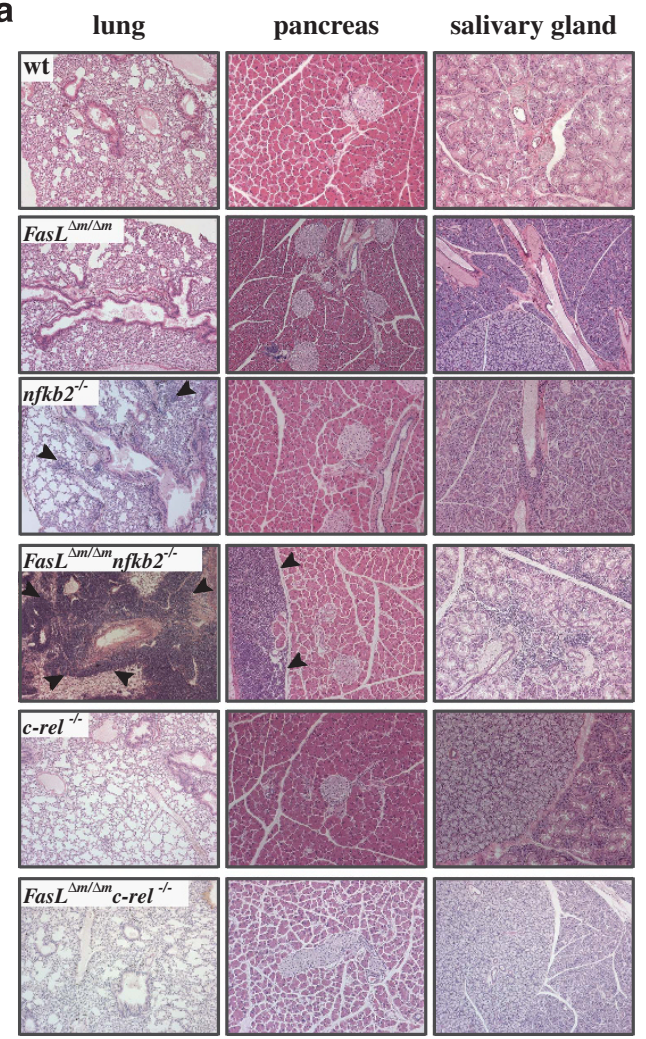

b

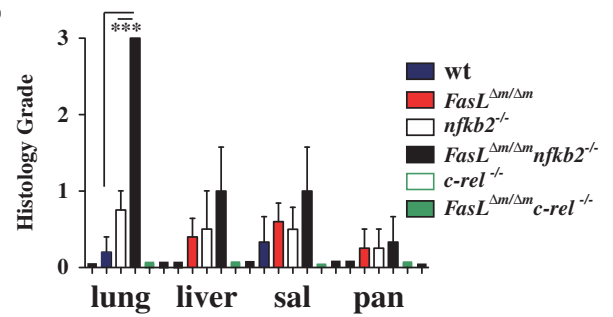

C

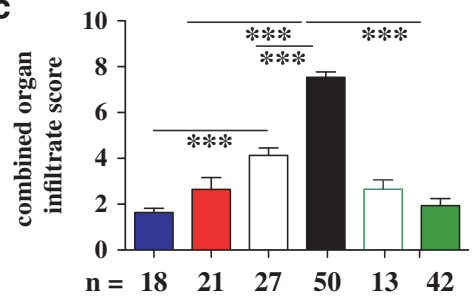

d

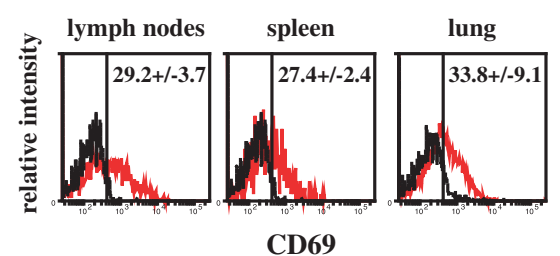

e

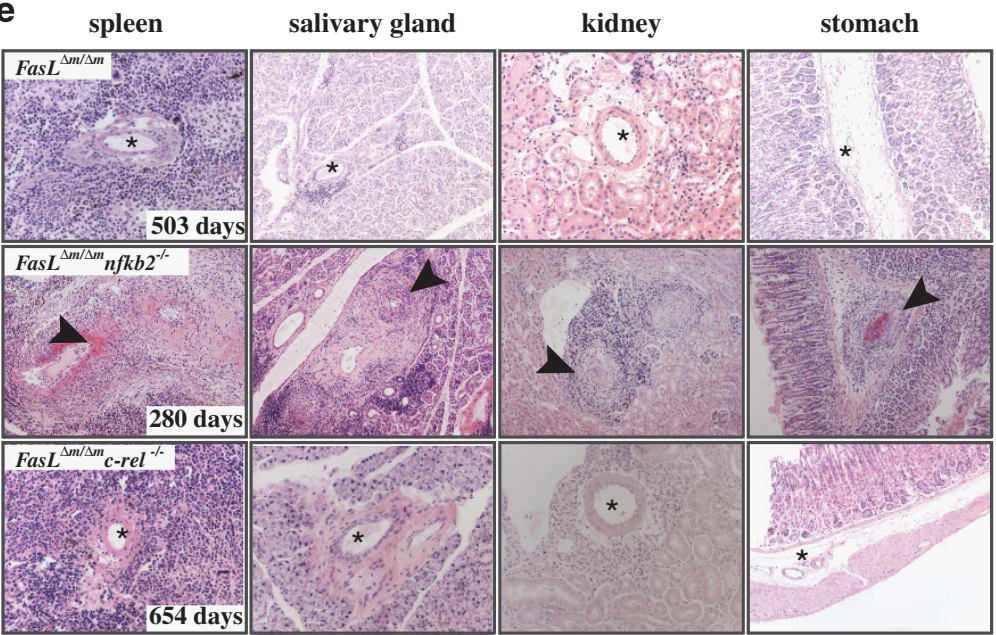

f
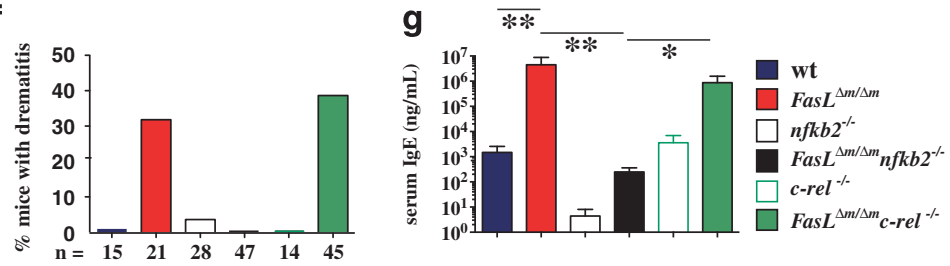

h

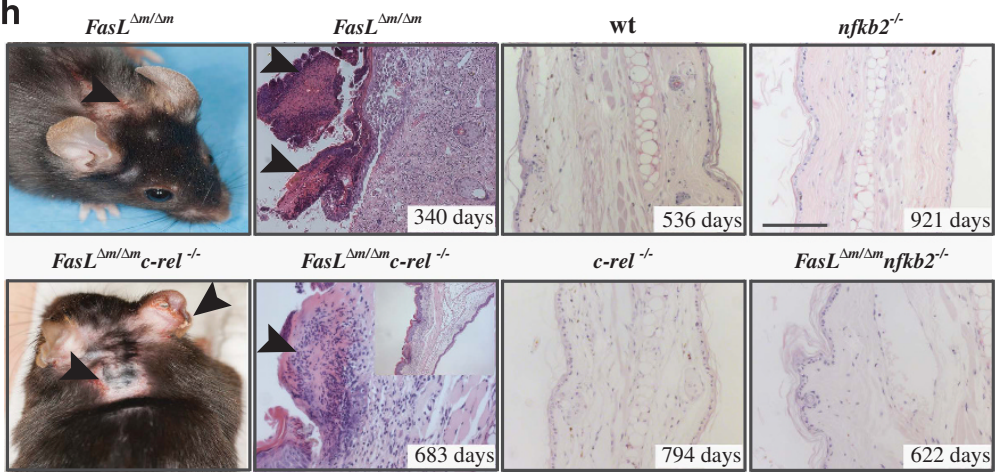

Figure 4 FasL ${ }^{\Delta m / \Delta m} n f k b 2^{-1-}$ mice developed more severe lymphoproliferative disease compared with FasL ${ }^{\Delta m / \Delta m}$ mice. (a) Representative photomicrographs of the lung, pancreas and salivary glands from mice of the indicated genotypes ( 5 months); arrows indicate areas of infiltration; $n=4 /$ genotype. (b) Summary (mean \pm S.E.M.) of the incidence of lymphocytic infiltration into the indicated organs of mice of the indicated genotypes (5 months of age), graded 0-3+, 3-5 mice/genotype; *** $P<0.0001$. (c) Graphical depiction (mean \pm S.E.M.) of the combined scores for lymphocytic infiltration (lung, liver, pancreas, salivary and stomach), each scored 0-3+ from the indicated mice when sick/

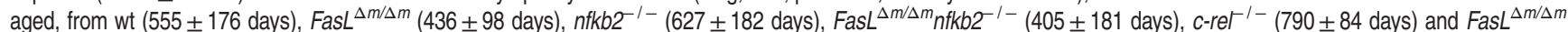
$c$-rel ${ }^{\prime-}$ (603 \pm 116 days) mice, ${ }^{* \star *} P<0.0001, n=$ number of mice analyzed. (d) Expression of the activation marker CD69 on CD4 ${ }^{+} \mathrm{T}$ cells (percentage of positive cells indicated) from the indicated organs from FasL ${ }^{\Delta m / \Delta m} n f k b 2^{-1-}$ mice (red line) and wt mice (black line); $n=3 /$ genotype. (e) Representative photomicrographs depicting necrotizing vasculitis in mice of the indicated genotypes. Arrows indicate areas of necrotizing vasculitis; *indicates vasculature. Magnification $\times 20$. (f) Graphical representation of the percentages of mice of the indicated genotypes with autoimmune dermatitis (ears and neck); $n=$ number of mice per genotype analyzed. (g) Serum lgE levels measured by ELISA when the animals were afflicted with dermatitis or otherwise sick. (h) Representative images, depicting autoimmune dermatitis either macroscopically or microscopically. Magnification $\times 10$ or $\times 40$; scale bar $128 \mu \mathrm{m}$ 

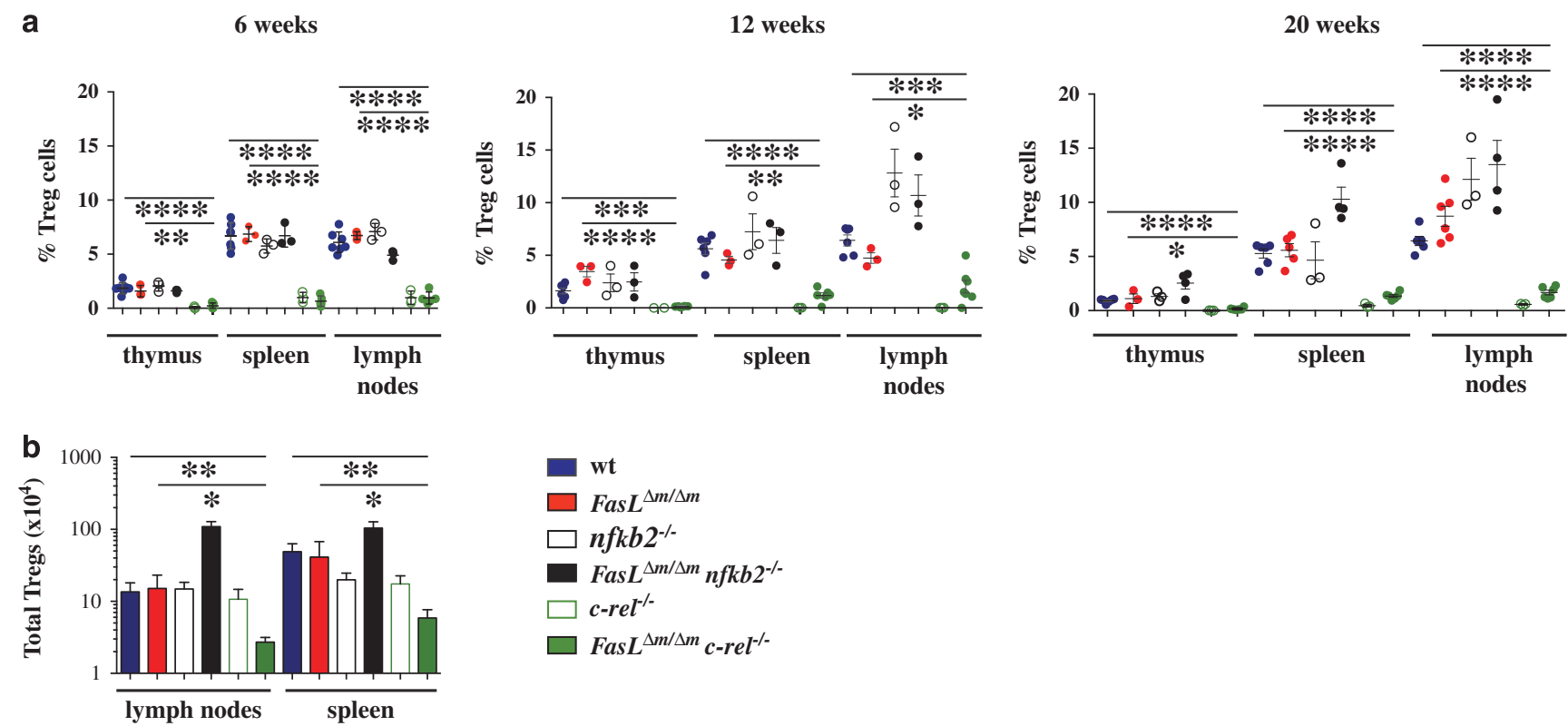

Figure $5 \mathrm{CD} 4^{+}$Tregs were not a limiting factor in the autoimmune disease of Fas $L^{\Delta m / \Delta m}$ mice. (a) The percentages of Treg $\left(C D 4^{+} C D 25^{+} F o x p 3^{+}\right)$were determined by flow cytometric analysis of the thymus, spleen and lymph nodes of mice of the indicated genotypes. Each dot represents a single mouse ( $n=3-4 /$ genotype); bars indicate the average. (b) Absolute numbers of Tregs $\left(\mathrm{CD}^{+} \mathrm{CD} 25^{+} \mathrm{Foxp}^{+}\right)$determined by flow cytometry $(n=4 /$ genotype at 3 months of age). The legend in $\mathbf{b}$ also applies to $\mathbf{a}$. Error bars represent S.E.M., ${ }^{*} P<0.05,{ }^{* *} P<0.001,{ }^{* \star *} P<0.0001$

Table 2 Summary of the effects that loss of NF-KB2 or c-REL exerts on abnormalities in FasL ${ }^{\Delta m / \Delta m}$ mice

\begin{tabular}{|c|c|c|c|c|c|c|c|}
\hline Genotype & Lifespan & $\begin{array}{l}\text { Cytokine } \\
\text { production }\end{array}$ & $\begin{array}{l}\text { Lymphadenopathy } \\
\text { splenomegaly }\end{array}$ & Organ infiltration & Serum IgG & ANA & Dermatitis \\
\hline $\begin{array}{l}\text { FasL }^{\Delta m / \Delta m^{\mathrm{a}}} \\
\text { FasL }^{\Delta m / \Delta m} n f k b 2^{-/-\mathrm{b}} \\
\text { FasL }^{\Delta m / \Delta m} c_{c-r e l^{-/-^{-}}}\end{array}$ & $\begin{array}{l}\text { Reduced } \\
\text { Minimal effect } \\
\text { Enhanced }\end{array}$ & $\begin{array}{l}\text { Elevated } \\
\text { No effect } \\
\text { Reduced }\end{array}$ & $\begin{array}{l}\text { Present } \\
\text { Accelerated } \\
\text { No effect }\end{array}$ & $\begin{array}{l}\text { Present } \\
\text { Accelerated } \\
\text { Reduced }\end{array}$ & $\begin{array}{l}\text { Elevated } \\
\text { Reduced } \\
\text { Reduced }\end{array}$ & $\begin{array}{l}\text { Present } \\
\text { Reduced } \\
\text { Reduced }\end{array}$ & $\begin{array}{l}\text { Present } \\
\text { Absent } \\
\text { Present }\end{array}$ \\
\hline
\end{tabular}

${ }^{\mathrm{a} C}$ Compared with wt. ${ }^{\mathrm{b}}$ Compared with $F a s L^{\Delta m / \Delta m}$

in $n f k b 2^{-/-}$mice ${ }^{18,26}$ and the exacerbated organ-specific lymphocyte infiltration observed in FasL ${ }^{\Delta m / \Delta m} n f k b 2^{-1-}$ animals.

\section{Discussion}

The many roles of canonical and non-canonical NF- $k B$ signaling in the immune system identify constituents of these pathways as potential therapeutic targets for inflammatory and autoimmune diseases. Indeed, the immunosuppressive and anti-inflammatory activity of glucocorticoids, a treatment of choice for many autoimmune diseases, is at least in part due to attenuated NF- $k B$ signaling. ${ }^{20}$ However, as NF- $k B$ signaling regulates a wide variety of biological processes both within and outside the immune system, prolonged blockade of overall NF- $k B$ activity, not surprisingly, can cause severe adverse effects. ${ }^{37}$ Accordingly, we assessed whether targeting individual NF-KB transcription factors would be a superior strategy for treating autoimmune disease. Fas $L^{\Delta m / \Delta m}$ mice were used as a model to determine the impact that the loss of c-REL (canonical pathway) or NF-kB2 (non-canonical pathway) had on their multifaceted autoimmune pathology. The largely hematopoietic restricted expression of $\mathrm{c}-\mathrm{REL}^{21}$ offers the added advantage of primarily targeting immune functions. ${ }^{22}$
Notably, targeting the canonical pathway in $T$ cells using a proteasome degradation resistant form of $\mid \mathrm{KB} a(\mathrm{I} \mathrm{KB})$ afforded only minor protection from autoimmune disease in Fas $L^{\text {gld/gld }}$ mice. ${ }^{38}$ However, given that NF-KB signaling was only diminished, not abrogated, combined with the essential loss of FAS function in B cells in this autoimmune disease, ${ }^{39}$ the full impact of completely inhibiting a particular NF-kB transcription factor on autoimmune disease caused by defects in FASL/FAS remains unclear. Our study is the first detailed, long-term analysis of the roles that specific NF- $k B$ family members have in the autoimmune disease caused by defects in FASL/FAS.

With respect to non-canonical NF- $k B$ signaling, loss of NF-kB2 had contrasting impacts on different aspects of the $\mathrm{FasL}^{\Delta m / \Delta m}$ autoimmune disease. Autoantibody-mediated pathologies (dermatitis and severe GN) were reduced but the lymphoproliferative disease was enhanced, causing respiratory difficulties and multiorgan vasculitis. This establishes that NF-kB2-dependent signals restrain proliferation and/or survival of the lymphocytes that accumulate to excess as a consequence of defective FASL/FAS-mediated apoptosis. Our studies using chimeric mice indicate that NF- $k$ B2 activity within the hematopoietic system is mainly responsible for restraining the proliferation and/or survival of these $T$ lymphocytes, although non-hematopoietic functions regulated 
a

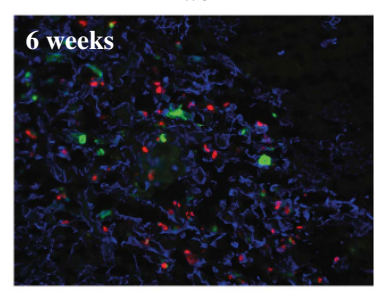

FasL $^{\Delta m / \Delta m}$

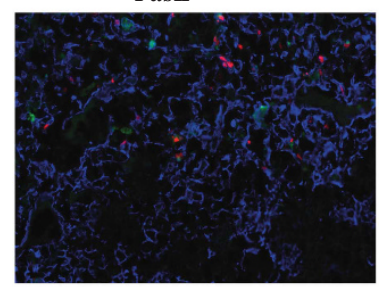

b

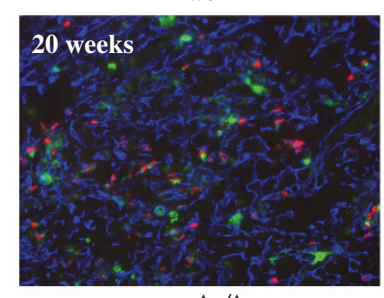

Fas L $^{\Delta m / \Delta m}$
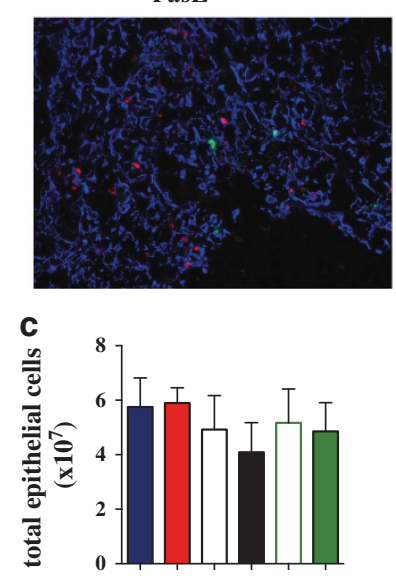

e

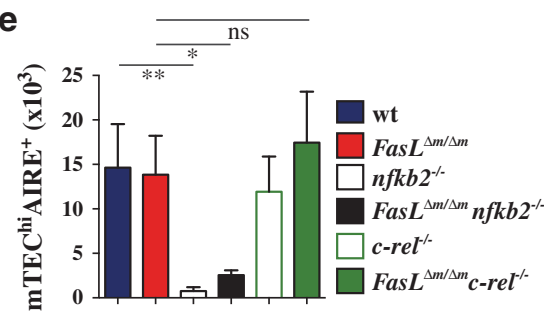

$n f k b 2^{-/-}$

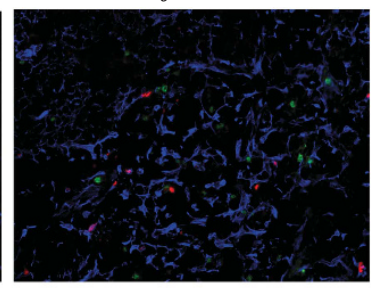

FasL $^{\Delta m / \Delta m} n f k b 2^{-/-}$

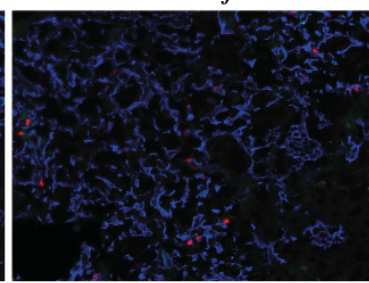

nfkb2 $2^{-/-}$

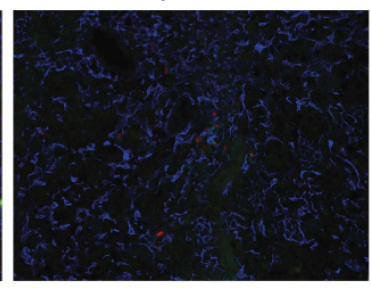

FasL $^{\Delta m / \Delta m} n f k b 2^{-/-}$

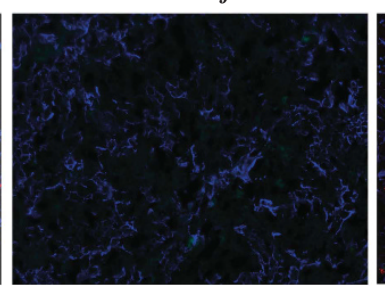

d $\quad 50, \frac{}{\mathrm{ns}}$

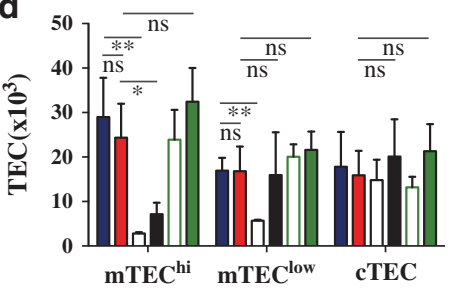

f

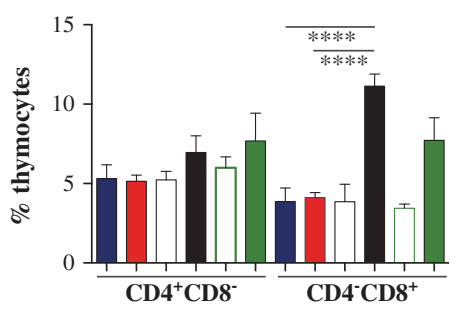

Figure 6 FasL ${ }^{\Delta m / \Delta m}$ mice had normal mTEC numbers with normal AIRE expression but loss of NF- $\kappa$ B2 caused abnormalities in the thymic stroma. (a) Representative confocal images of thymi from mice of the indicated genotypes at 6 weeks. Sections were stained for UEA-1 (green), AIRE (red) and keratin-5 (blue); $n=3 /$ genotype/time point. (b) Representative confocal images of thymi from mice of the indicated genotypes at 20 weeks as for (a). (c) Graphical representation of total thymic cellularity from mice of the indicated genotypes at 20 weeks of age; $n=4 /$ genotype. (d) Graphical depiction of flow cytometric analysis of TECs from mice of the indicated genotypes at 20 weeks of age, as

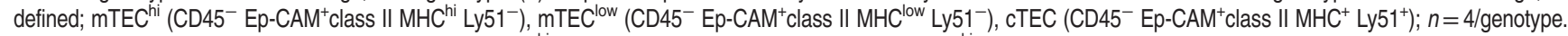

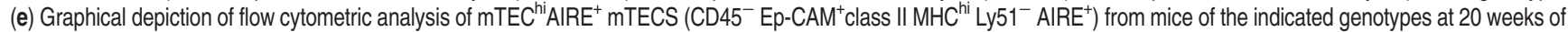
age. (f) Percentages of thymocytes that are $\mathrm{CD} 4$ single positive $\left(\mathrm{CD} 4^{+} \mathrm{CD} 8^{-}\right)$or $\mathrm{CD} 8$ single positive $\left(\mathrm{CD} 4^{-} \mathrm{CD} 8^{+}\right)$from mice of the indicated genotypes at 3 months of age; $n=4 /$ genotype. ${ }^{*} P<0.05,{ }^{\star *} P<0.005$ 
by NF- $\kappa \mathrm{B} 2$ may contribute. The NF- $\kappa \mathrm{B} 2$ precursor p100, a protein with $I_{\kappa} \mathrm{B}$-like activity, ${ }^{40}$ limits NF- $\kappa \mathrm{B}$-dependent T-cell proliferation by preventing the nuclear activation of p50-NF$\kappa \mathrm{B} 1 / \mathrm{p} 65$ heterodimers. ${ }^{41}$ The absence of this inhibitory $\mathrm{p} 100$ NF- $k$ B2 protein may be a mechanism that contributes to the increased accumulation of T cells in Fas $L^{\Delta m / \Delta m} n f k b 2^{-1-}$ mice. The escape of $T$ cells with higher affinity autoreactive TCRs due to impaired negative selection as a consequence of reduced numbers of $\mathrm{AIRE}^{+}$mTECS might also contribute to the enhanced accumulation of DN T cells in FasL ${ }^{\Delta m / \Delta m} n f k b 2^{-1-}$ mice. Finally, the reduction in autoantibody-mediated aspects of FasL ${ }^{\Delta m / \Delta m}$ autoimmune disease associated with NF- $\kappa$ B2 loss can be explained by reduced BAFF-R survival signals in $B$ cells and/or germinal center defects, both of which involve p52-NF- $k$ B2/RELB dimers. ${ }^{19}$ Overall, these findings suggest that, while systemic therapeutic targeting of NF-KB2 could alleviate autoantibody-induced pathology, this benefit would be negated by enhanced lymphoproliferative disease.

Our study also provides insight into the role of NF- $\kappa$ B2 in autoimmune dermatitis. Unlike Fas $L^{\Delta m / \Delta m}$ mice that develop IgE-dependent autoimmune dermatitis, the FasL ${ }^{\Delta m / \Delta m} n f k b 2^{-/-}$ mice were protected. Consistent with NIK, the apical kinase in the non-canonical NF-KB2-dependent pathway ${ }^{42}$ being critical for isotype switching to $\operatorname{lgE},{ }^{42}$ our aged $n f k b 2^{-1-}$ mice had $\sim 100$-fold lower serum IgE levels compared with wt controls, and even in FasL ${ }^{\Delta m / \Delta m} n f k b 2^{-1-}$ mice the serum IgE levels were still below those found in wt mice. Given that topical delivery of NF- $\kappa$ B decoy oligodeoxynuclotides alleviates murine atopic dermatitis, ${ }^{43}$ our findings indicate that topical NF- $k \mathrm{~B} 2$ inhibitors may represent a promising strategy for treating IgE-dependent autoimmune dermatitis. In contrast to a prior report, which showed that isotype switching to $\lg \mathrm{E}$ was $\mathrm{C}-\mathrm{REL}$ dependent, ${ }^{44,45}$ we found that IgE levels were not markedly reduced by loss of $c-R E L$ on either a wt or a Fas $L^{\Delta m / \Delta m}$ background. The reason for these differences remains to be determined.

This report demonstrates that c-REL loss substantially prolongs the lifespan of $\mathrm{FasL}^{\Delta m / \Delta m}$ mice by preventing autoantibody-mediated pathology. Although $c-R E L$ is critical for normal T-cell activation through a combination of T-cell intrinsic and extrinsic processes, ${ }^{12,22,28}$ lymphadenopathy was unaffected by loss of $c-R E L$. This indicates that the accumulation of DN T cells is driven by other NF- $k$ B proteins or is NF- $\kappa \mathrm{B}$ independent. This disjunction between extended lifespan despite persistence of the lymphoproliferative component of the autoimmune disease elicited by defects in FASL/ FAS is consistent with studies of gene-targeted mice lacking FAS in select cell populations. ${ }^{39}$ Loss of c-REL has also been shown to prevent pathology in mouse models of collageninduced arthritis, ${ }^{46}$ streptozotocin-induced diabetes ${ }^{47}$ and autoimmune encephalomyelitis. ${ }^{48}$ These outcomes can be explained by the involvement of Th1 cytokines and/or IL-17 in these diseases, because their production and the differentiation of Th1, ${ }^{49}$ as well as Th $17^{48}$ effector T cells, are c-RELdependent. The fact that $\mathrm{c}-\mathrm{REL}$ is also critical for cytokine production by macrophages, dendritic cells and NKT cells ${ }^{12}$ is also likely to contribute to the reduced pathology in Fas ${ }^{\Delta m / \Delta m}$ $c-r e I^{\prime-}$ mice. Although we were unable to detect IFN $\beta$ in the sera of sick FasL ${ }^{\Delta m / \Delta m}$, FasL ${ }^{\Delta m / \Delta m} n f k b 2^{-1-}$ and FasL ${ }^{\Delta m / \Delta m}$ $c-\mathrm{rel}^{\prime-}$ mice, others have reported that introduction of a loss-of-function mutation of the type I interferon receptor decreased glomerular immune complex deposition in Fas ${ }^{\text {Ipr/lpr }}$ mutant mice. ${ }^{27}$ However, no data on animal lifespan were provided and these mice were on a mixed genetic background; notably, genetic background greatly affects pathology in Fas ${ }^{\text {Ipr/pr }}$ mice. Thus, the role of type I interferons in SLE-like disease in mice with defects in FASL or FAS remains unclear.

$A$ requirement for $c-R E L$ in antigen receptor-induced $T-$ and B-cell activation ${ }^{23}$ is the most likely explanation for why the reduction in Treg numbers, caused by the loss of $c-R E L,{ }^{33}$

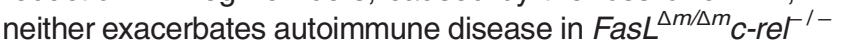
mice nor instigates autoimmunity in $\mathrm{C}^{-\mathrm{re}^{\prime}}{ }^{\prime-}$ mice. ${ }^{23}$ Interestingly, mice deficient for PELI1, ${ }^{50}$ a unbiquitin E3-ligase that controls c-REL levels, develop an autoimmune phenotype similar to those observed in FasL ${ }^{\Delta m / \Delta m}$ mice, accompanied by nuclear accumulation of $c-R E L$ and excessive canonical NF- $\kappa B$ activation. As PELI1 appears to be critical for the maintenance of T-cell tolerance, it would be interesting to test whether c-REL dysregulation, specifically in T cells, is responsible for the SLElike autoimmune disease in FasL ${ }^{\Delta m / \Delta m}$ mice.

In conclusion, our studies using FasL ${ }^{\Delta m / \Delta m}$ mice demonstrate that the canonical and non-canonical NF- $\kappa$ B pathways have critical, yet distinct roles in autoimmune and lymphoproliferative pathology. Although autoimmune diseases caused by inherited mutations in FASL or FAS (ALPS patients) are rare, subtle abnormalities in this apoptotic pathway arising from gene polymorphisms are likely to contribute to autoimmune disease predisposition more generally. ${ }^{51}$ Combined with the recent discovery of links between polymorphisms in $c-R e l$ and increased susceptibility to certain human autoimmune diseases, ${ }^{23}$ our findings suggest that targeting c-REL constitutes an attractive strategy to alleviate or prevent autoimmune pathology, while avoiding the major side effects associated with pan-NF- $\kappa \mathrm{B}$ inhibition.

\section{Materials and Methods}

Mice. All animal experiments were approved by the Walter and Eliza Hall Institute Animal Ethics Committee. FasL ${ }^{\Delta m / \Delta m}$ mice were generated on a C56BL/6 genetic background. $^{8}$ nfkb2 $2^{-1-25}$ and $\mathrm{c}^{-\mathrm{reF}^{\prime-}}$ mice $^{22,28}$ were generated on a mixed C57BL/6x129SV background and backcrossed onto a C57BL/6 background for $>10$ generations. FasL ${ }^{\Delta \mathrm{m} / \Delta \mathrm{m}}$ mice were intercrossed with $n \mathrm{fkb2}^{-1-}$ or $\mathrm{c}-\mathrm{rel}^{-1-}$

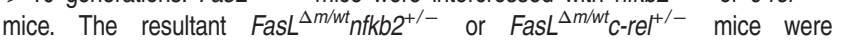
intercrossed to produce FasL ${ }^{\Delta m / \Delta m}$ nfkb2 $^{-1}$ or FasL ${ }^{\Delta m / \Delta m} \mathrm{C}^{-r e l^{\prime-}}$ - mice. Control wt, FasL ${ }^{\Delta m / \Delta m}, n f k b 2^{-1-}$ and $c-r e l^{\prime-}$ mice used in this study were maintained within the same colony.

Histological analysis and scoring. Mice were killed by $\mathrm{CO}_{2}$ asphyxiation. Organs were removed and fixed in $80 \%$ Histochoice (Amresco, Solon, OH, USA) or $10 \%$ buffered formalin and paraffin embedded. Histological examination was performed by staining with hematoxylin and eosin $(\mathrm{H} \& \mathrm{E})$. H\&E-stained sections of the kidneys were examined for evidence of GN and scored on a scale of 0-4 $(0=$ normal; $1=$ minor mesangial hypercellularity; $2=$ moderate hypercellularity; 3 =severe hypercellularity; $4=$ severe hypercellularity with obliteration and thickening of all capillary loops or the presence of fibrinoid necrosis or glomerular crescents). Lymphoid infiltration into organs (lung, liver, pancreas, submandibular gland and stomach) was assessed by examining H\&E-stained sections and graded 0-3 $(0=$ none; $1=$ occasional small perivascular foci (age related); $2=$ more dense, well-defined perivascular and periductal foci; $3=$ extensive infiltrate with parenchymal destruction). Organs were also examined and scored for the presence of necrotizing vasculitis. All photomicrographs were acquired using a $\times 10 / \mathrm{NA} 0.3$, $\times 20 / \mathrm{NA} 0.50$ or $\times 40 / \mathrm{NA} 0.75$ objective lens attached to an Axioplan 2 microscope (Carl Zeiss, North Ryde, NSW, Australia). 
Immunofluorescent staining and confocal microscopy. The presence of autoantibodies against classical target tissues in autoimmune diseases (salivary gland, lacrimal gland, eye, kidney, liver, lung, pancreas, stomach and thyroid) was assessed by staining frozen tissue sections ( $5 \mu \mathrm{m}$, air dried and fixed in acetone) of rag- $1^{-1-}$ mice (to eliminate endogenous $\mathrm{lg}$, a source of background staining) with sera (1/100 dilution) from aged or sick mice. Staining was detected with FITC conjugated goat antibodies against mouse IgG, IgA or IgM (Cappel (MP Biochemicals), Santa Ana, CA, USA). To stain for immune complex deposits, the kidneys from aged or sick mice were snap-frozen in isopentane, sectioned, acetone fixed, and blocked with PBS/2\% FCS, followed by staining with FITC-coupled goat antibodies specific to mouse IgM, IgG or IgA (Southern Biotechnology, Birmingham, AL, USA). All immunofluorescent staining was analyzed using a Leica laser scanning (SP2) confocal microscope.

Frozen sections of thymi from 6 - or 20 -week-old mice were cut at $-20^{\circ} \mathrm{C}$ on a cryostat $(5 \mu \mathrm{m})$, air dried and fixed in acetone. Sections were incubated with primary $\mathrm{mAbs}$ in $0.5 \% \mathrm{FCS} / \mathrm{PBS}$ for $30 \mathrm{~min}$ in a moist chamber, washed three times in PBS and incubated, while protected from light, with the appropriate secondary Abs plus DAPI, followed by washing. Primary Abs included biotin-conjugated anti-UEA-1 (Vector Laboratories, Burlingame, CA, USA), rabbit anti-keratin-5 (MK5, Covance, Biolegend, San Deigo, CA, USA) and Alexa 405-conjugated anti-AIRE. Detection was achieved using Alexa Fluor 555-conjugated goat anti-rabbit IgG antibodies or FITCconjugated Streptavidin (both from Invitrogen, Carlsbad, CA, USA). Staining was analyzed using a Zeiss LSM5 line scanning confocal microscope (Carl Zeiss).

ANA in sera from aged or sick mice was detected by staining (1/100 serum dilution) of slides coated with HEp-2 human epithelial cells according to the manufacturer's instructions (Immuno Concepts, Sacramento, CA, USA) and detected with FITCconjugated rabbit antibodies against all mouse Igs (Cappel (MP Biochemicals)) or FITC-conjugated rabbit antibodies selective for mouse $\lg M$, IgG or $\lg A$ (Southern Biotechnology). ANA levels were quantified according to the brightness of fluorescence intensity on a scale of 0 (no fluorescence) to 3+ (maximum fluorescence intensity). Serum ANA levels were also measured by ELISA (The Binding, Site, Birmingham, UK) according to the manufacturer's instructions.

Hematopoietic reconstitution. Ablation of the hematopoietic compartment was achieved by exposing mice to two doses of $5.5 \mathrm{~Gy}$ of $\gamma$-irradiation from a ${ }^{60} \mathrm{Co}$ source, $2 \mathrm{~h}$ apart. Irradiated mice were reconstituted by intravenous (i.v.) injection of $10^{6}$ total bone marrow cells in a $100 \mu$ l volume of PBS. Reconstituted mice were maintained on $2 \mathrm{mg} / \mathrm{ml}$ neomycin-supplemented water for 21 days.

Flow cytometric analysis of thymic stromal cell populations. This procedure was adapted from previously described protocols. ${ }^{52,53}$ Thymi were processed individually for digestion as described. ${ }^{53}$ First, thymi were cleaned of fat and connective tissue, the lobes separated and the capsule nicked with fine scissors. Thymi were then individually agitated in $10 \mathrm{ml} \mathrm{RPMI-1640} \mathrm{medium} \mathrm{for}$ several minutes to gently flush out thymocytes using a wide-bore pipette. The remaining thymic tissue was then incubated at $37^{\circ} \mathrm{C}$ for $15 \mathrm{~min}$ in $1 \mathrm{ml}$ of Liberase/ DNAase I ( 0.5 units and $0.1 \%$, respectively, both from Roche, Germany) in RPMI-1640 medium with regular agitation, as detailed. ${ }^{54}$ Fragments were allowed to settle and the supernatant was collected (fraction 1), kept on ice and the digestion process repeated with the settled thymic fragments. After a second digestion (fraction 2), the remaining aggregates were centrifuged, and resuspended in PBS with $5 \mathrm{mM} \mathrm{EDTA}, 2 \% \mathrm{FCS}$ and $0.02 \% \mathrm{NaN}_{3}$. Cells were then filtered through a $100 \mathrm{~mm}$ mesh and counted using a Countless automated cell counter (Invitrogen). For phenotypic analysis, $1.5 \times 10^{7}$ cells were stained.

Flow cytometric analysis. Single-cell suspensions of the spleen and lymph nodes (pooled axillary, brachial, inguinal and mesenteric) were stained as described $^{1}$ with FITC-, R-PE- or APC-conjugated mAbs specific for mouse cell surface markers: RB6-8C5: anti-Gr-1, MI/70: anti-Mac-1, M3/84.6.34: anti-Mac-2, Ter119, F4/80, T24.31.2: anti-Thy-1, GK1.5: anti-CD4, 53.6.72: anti-CD8, RA3-6B2: anti-CD45R-B220 and PK136: anti-NK1.1 (BD Biosciences, Franklin Lakes, NJ, USA). The vital dye $\mathrm{PI}(1 \mu \mathrm{g} / \mathrm{ml})$ was included and data were acquired on a FACScan (Becton Dickinson, Franklin Lakes, NJ, USA) or LSR II (Becton Dickinson). Tregs were detected after fixation and permeabilization (eBiosciences) by staining with anti-CD25-R-PE, anti-CD4-FITC and anti-Foxp3-APC antibodies, followed by data acquisition on a LSR II (Becton Dickson). Single-cell suspensions were prepared from the lungs by digestion for $20 \mathrm{~min}$ at room temperature with collagenase/DNAse (7 mg collagenase (Worthington, Lakewood, NJ, USA) in $1 \mathrm{ml}$ volume of $0.1 \%$ w/v DNAse1 (Roche)) treated with EDTA as described, ${ }^{55}$ and then stained with an R-PE-coupled antibody to Thy-1 plus a panel of biotinylated antibodies specific to different $\operatorname{TCRV} \beta$ regions followed by staining with FITCcoupled streptavidin (BD-Pharmingen).

Phenotypic analysis of thymic stromal cells by flow cytometry was performed using the following antibody conjugates: PerCP-cy5.5 anti-CD45.2 (clone 30F11, Biolegend, San Diego, CA, USA) and PE anti-Ly51 (Biolegend). APC anti-AIRE, FITC anti-MHC class II (H-2A/E, clone M5 114/15.2) and APC-cy7 anti-EpCAM (clone G8.8a) were produced in-house.

Measurement of serum immunoglobulin levels. Serum immunoglobulin levels were determined by ELISA using sheep anti-mouse Ig antibodies (Silenus Laboratories, Australia) as a capture reagent and developed with mouse Ig isotypespecific goat antibodies conjugated to horseradish peroxidase (Southern Biotechnology). Purified myeloma proteins were used as standards (Sigma, MO, USA).

Analysis of serum cytokine and chemokine levels. Cytokine and chemokine levels in sera were measured by using Bio-Plex Pro mouse cytokine 23-plex (Bio-Rad, Gladesville, NSW, Australia) and Bio-Plex Pro mouse cytokine Th17-plex magnetic bead immunoassays on a Bio-Rad Bio-Plex instrument, following the manufacturer's instructions. The cytokines measured included the following: CD40L, IL-1 $\alpha$, IL- $\beta$, IL-2, IL-3, IL-4, IL-5, IL-6, IL-9, IL-10, IL-12(P40), IL-12(p70), IL-13, IL-17A/F, IL-21, IL-22, IL-23(p19), IL-31, IL-33, eotaxin, G-CSF, GM-CSF, IFN- $\gamma$, KC (CXCL1), MCP-1, MIP- $1 \alpha$, MIP- $1 \beta$, MIP- $3 \alpha$, RANTES and $\operatorname{TNF} \alpha$. Serum IFN $\beta$ levels were measured using the VeriKine-HS (PBL Assay Science, Piscataway, NJ, USA) Mouse IFN- $\beta$ serum ELISA kit, according to the manufacturer's instructions.

Statistical analysis. Statistical analysis was performed using Student's t-test, log rank (Mantel-Cox) test for animal survival curves, one-way analysis of variance using Tukey's comparison test to compare multiple groups or the Mann-Whitney test where appropriate.

\section{Conflict of Interest}

The authors declare no conflict of interest.

Acknowledgements. We thank G Siciliano, K Hughes, J Coughlin, K McKenzie and F Dabrowski, for animal care; J Corbin for automated blood analysis; B Helbert and $\mathrm{C}$ Young for genotyping; and $\mathrm{E}$ Tsui, V Babo, K Weston and all histology staff for preparation of histological sections. This work was supported by fellowships and grants from the NHMRC (Canberra; programs \#461221 and \#1016701, fellowship; (DG) \#637353 and project grants \#1009145 (LOR), \#637332 (DG) and an NHMRC infrastructure grant, Independent Research Institutes Infrastructure Support Scheme Grant \#361646, the Victorian State Government (OIS grant), the Leukemia and Lymphoma Society (SCOR grant \#7413 and \#7001-13) and the JDRF/NHMRC \#466658 (AS). This research was also supported in part by the Intramural Research Program of the National Institute of Allergy and Infectious Diseases, National Institutes of Health (US).

1. Strasser A, Harris AW, Cory S. Bcl-2 transgene inhibits T cell death and perturbs thymic selfcensorship. Cell 1991; 67: 889-899.

2. Goodnow CC, Sprent J, Fazekas de St Groth B, Vinuesa CG. Cellular and genetic mechanisms of self tolerance and autoimmunity. Nature 2005; 435: 590-597.

3. Bouillet P, Purton JF, Godfrey DI, Zhang L-C, Coultas L, Puthalakath $\mathrm{H}$ et al. BH3-only Bcl-2 family member Bim is required for apoptosis of autoreactive thymocytes. Nature 2002; 415 : 922-926.

4. Strasser A, Jost PJ, Nagata S. The many roles of FAS receptor signaling in the immune system. Immunity 2009; 30: 180-192.

5. Hughes PD, Belz GT, Fortner K, Budd RC, Strasser A, Bouillet P. Apoptosis regulators Fas and Bim cooperate in shutdown of chronic immune responses and prevention of autoimmunity. Immunity 2008; 28: 197-205.

6. Watanabe-Fukunaga R, Brannan $\mathrm{Cl}$, Copeland NG, Jenkins NA, Nagata S. Lymphoproliferation disorder in mice explained by defects in Fas antigen that mediates apoptosis. Nature 1992; 356: 314-317.

7. Fisher GH, Rosenberg FJ, Straus SE, Dale JK, Middelton LA, Lin AY et al. Dominant interfering Fas gene mutations impair apoptosis in a human autoimmune lymphoproliferative syndrome. Cell 1995; 81: 935-946.

8. O'Reilly LA, Tai L, Lee L, Kruse EA, Grabow S, Fairlie WD et al. Membrane-bound Fas ligand only is essential for Fas-induced apoptosis. Nature 2009; 461: 659-663.

9. Krammer PH. CD95's deadly mission in the immune system. Nature 2000; 407: 789-795. 
10. Ahn JH, Park SM, Cho HS, Lee MS, Yoon JB, Vilcek J et al. Non-apoptotic signaling pathways activated by soluble Fas ligand in serum-starved human fibroblasts. Mitogenactivated protein kinases and NF-kappaB-dependent gene expression. J Biol Chem 2001; 276: 47100-47106.

11. Karin M, Lin A. NF- $\kappa B$ at the crossroads of life and death. Nat Immunol 2002; 3: 221-227.

12. Gerondakis S, Grumont R, Gugasyan R, Wong L, Isomura I, Ho W et al. Unravelling the complexities of the NF-kappaB signalling pathway using mouse knockout and transgenic models. Oncogene 2006; 25: 6781-6799.

13. Hayden MS, Ghosh S. Shared principles in NF-kappaB signaling. Cell 2008; 132: 344-362.

14. Gilmore TD. Introduction to NF-kappaB: players, pathways, perspectives. Oncogene 2006; 25: $6680-6684$.

15. Vallabhapurapu S, Karin M. Regulation and function of NF-kappaB transcription factors in the immune system. Annu Rev Immunol 2009; 27: 693-733.

16. Brown KD, Claudio E, Siebenlist U. The roles of the classical and alternative nuclear factorkappaB pathways: potential implications for autoimmunity and rheumatoid arthritis. Arthritis Res Ther 2008; 10: 212.

17. Pai S, Thomas R. Immune deficiency or hyperactivity-Nf-kappab illuminates autoimmunity. $J$ Autoimmun 2008; 31: 245-251.

18. Zhang B, Wang Z, Ding J, Peterson P, Gunning WT, Ding HF. NF-kappaB2 is required for the control of autoimmunity by regulating the development of medullary thymic epithelial cells. J Biol Chem 2006; 281: 38617-38624.

19. Siebenlist U, Brown K, Claudio E. Control of lymphocyte development by nuclear factor-kappaB. Nat Rev Immunol 2005; 5: 435-445.

20. Flammer JR, Rogatsky I. Minireview: Glucocorticoids in autoimmunity: unexpected targets and mechanisms. Mol Endocrinol 2011; 25: 1075-1086.

21. Grumont RJ, Gerondakis S. The murine c-rel proto-oncogene encodes two mRNAs the expression of which is modulated by lymphoid stimuli. Oncogene Res 1990; 5: 245-254.

22. Gerondakis S, Strasser A, Metcalf D, Grigoriadis G, Scheerlinck J-PY, Grumont RJ. Rel-deficient $T$ cells exhibit defects in production of interleukin 3 and granulocytemacrophage colony-stimulating factor. Proc Natl Acad Sci USA 1996; 93: 3405-3409.

23. Gilmore TD, Gerondakis S. The c-Rel transcription factor in development and disease. Genes Cancer 2011; 2: 695-711.

24. Caamaño JH, Rizzo CA, Durham SK, Barton DS, Raventós-Suárez C, Snapper CM et al. Nuclear factor (NF)- $\mathrm{B}$ B2 (p100/p52) is required for normal splenic microarchitecture and B cell-mediated immune responses. J Exp Med 998; 87: 85-96.

25. Franzoso G, Carlson L, Poljak L, Shores EW, Epstein S, Leonardi A et al. Mice deficient in nuclear factor (NF)- $\kappa \mathrm{B} / \mathrm{p} 52$ present with defects in humoral responses, germinal center reactions, and splenic microarchitecture. J Exp Med 998; 87: 47-59.

26. Zhu M, Chin RK, Christiansen PA, Lo JC, Liu X, Ware C et al. NF-kappaB2 is required for the establishment of central tolerance through an Aire-dependent pathway. J Clin Invest 2006; 116: 2964-2971.

27. Braun D, Geraldes P, Demengeot J. Type I Interferon controls the onset and severity of autoimmune manifestations in Ipr mice. J Autoimmun 2003; 20: 15-25.

28. Köntgen F, Grumont RJ, Strasser A, Metcalf D, Li R, Tarlinton D et al. Mice lacking the c-rel proto-oncogene exhibit defects in lymphocyte proliferation, humoral immunity, and interleukin-2 expression. Genes Dev 1995; 9: 1965-1977.

29. Werfel $T$. The role of leukocytes, keratinocytes, and allergen-specific $\lg E$ in the development of atopic dermatitis. J Invest Dermatol 2009; 129: 1878-1891.

30. Wing K, Sakaguchi S. Regulatory T cells exert checks and balances on self tolerance and autoimmunity. Nat Immunol 2010; 11: 7-13.

31. Akdis M, Blaser K, Akdis CA. T regulatory cells in allergy: novel concepts in the pathogenesis, prevention, and treatment of allergic diseases. J Allergy Clin Immunol 2005; 116: 961-968 quiz 969.

32. Fyhrquist N, Lehtimaki S, Lahl K, Savinko T, Lappetelainen AM, Sparwasser T et al. Foxp3+ cells control Th2 responses in a murine model of atopic dermatitis. J Invest Dermatol 2012; 132: $1672-1680$.
33. Isomura I, Palmer S, Grumont RJ, Bunting K, Hoyne G, Wilkinson N et al. c-Rel is required for the development of thymic Foxp3+ CD4 regulatory T cells. J Exp Med 2009; 206: 3001-3014.

34. Mathis D, Benoist C. Aire. Annu Rev Immunol 2009; 27: 287-312.

35. Gray DH, Gavanescu I, Benoist C, Mathis D. Danger-free autoimmune disease in Aire-deficient mice. Proc Natl Acad Sci USA 2007; 104: 18193-18198.

36. Irla M, Hollander G, Reith W. Control of central self-tolerance induction by autoreactive CD4 (+) thymocytes. Trends Immunol 2010; 31: 71-79.

37. Strnad J, Burke JR. IkappaB kinase inhibitors for treating autoimmune and inflammatory disorders: potential and challenges. Trends Pharmacol Sci 2007; 28: 142-148.

38. Vallabhapurapu S, Ryseck RP, Malewicz M, Weih DS, Weih F. Inhibition of NF- $\kappa$ B in T cells blocks lymphoproliferation and partially rescues autoimmune disease in gld/gld mice. Eur $J$ Immunol 2001; 31: 2612-2622.

39. Hao Z, Duncan GS, Seagal J, Su YW, Hong C, Haight J et al. Fas receptor expression in germinal-center B cells is essential for Tand B lymphocyte homeostasis. Immunity 2008; 29: 615-627.

40. Basak S, Kim H, Kearns JD, Tergaonkar V, O'Dea E, Werner SL et al. A fourth IkappaB protein within the NF-kappaB signaling module. Cell 2007; 128: 369-381.

41. Ishimaru N, Kishimoto H, Hayashi $Y$, Sprent J. Regulation of naive T cell function by the NF-kappaB2 pathway. Nat Immunol 2006; 7: 763-772.

42. Brady K, Fitzgerald S, Moynagh PN. Tumour-necrosis-factor-receptor-associated factor 6 , NF-kappaB-inducing kinase and lkappaB kinases mediate lgE isotype switching in response to CD40. Biochem J 2000; 350: 735-740.

43. Nakamura H, Aoki M, Tamai K, Oishi M, Ogihara T, Kaneda $Y$ et al. Prevention and regression of atopic dermatitis by ointment containing NF-kB decoy oligodeoxynucleotides in $\mathrm{NC} / \mathrm{Nga}$ atopic mouse model. Gene Ther 2002; 9: 1221-1229.

44. Zelazowski $\mathrm{P}$, Shen $\mathrm{Y}$, Snapper CM. NF-kappaB/p50 and NF-kappaB/c-Rel differentially regulate the activity of the 3 'alphaE-hsl,2 enhancer in normal murine $B$ cells in an activationdependent manner. Int Immunol 2000; 12: 1167-1172.

45. Carrasco D, Cheng J, Lewin A, Warr G, Yang H, Rizzo C et al. Multiple hemopoietic defects and lymphoid hyperplasia in mice lacking the transcriptional activation domain of the c-Rel protein. J Exp Med 1998; 187: 973-984

46. Campbell IK, Gerondakis S, O'Donnell K, Wicks IP. Distinct roles for the NF-kappaB1 (p50) and c-Rel transcription factors in inflammatory arthritis. $J$ Clin Invest 2000; 105: 1799-1806.

47. Lamhamedi-Cherradi SE, Zheng S, Hilliard BA, Xu L, Sun J, Alsheadat S et al. Transcriptional regulation of type I diabetes by NF-kappa B. J Immunol 2003; 171: 4886-4892.

48. Chen G, Hardy K, Pagler E, Ma L, Lee S, Gerondakis S et al. The NF-kappaB transcription factor c-Rel is required for Th17 effector cell development in experimental autoimmune encephalomyelitis. J Immunol 2011; 187: 4483-4491.

49. Hilliard BA, Mason N, Xu L, Sun J, Lamhamedi-Cherradi SE, Liou HC et al. Critical roles of c-Rel in autoimmune inflammation and helper T cell differentiation. J Clin Invest 2002; 110: 843-850.

50. Chang M, Jin W, Chang JH, Xiao Y, Brittain GC, Yu J et al. The ubiquitin ligase Peli1 negatively regulates $T$ cell activation and prevents autoimmunity. Nat Immunol 2011; 12: $1002-1009$.

51. Goodnow CC. Multistep pathogenesis of autoimmune disease. Cell 2007; 130: 25-35.

52. Gray DH, Chidgey AP, Boyd RL. Analysis of thymic stromal cell populations using flow cytometry. J Immunol Methods 2002; 260: 15-28.

53. Gray DH, Fletcher AL, Hammett M, Seach N, Ueno T, Young LF et al. Unbiased analysis, enrichment and purification of thymic stromal cells. J Immunol Methods 2008; 329: $56-66$.

54. Seach N, Wong K, Hammett M, Boyd RL, Chidgey AP. Purified enzymes improve isolation and characterization of the adult thymic epithelium. J Immunol Methods 2012; 385: 23-34.

55. Naik S, Vremec D, Wu L, O'Keeffe M, Shortman K. CD8alpha+ mouse spleen dendritic cells do not originate from the CD8alpha- dendritic cell subset. Blood 2003; 102: 601-604.

Supplementary Information accompanies this paper on Cell Death and Differentiation website (http://www.nature.com/cdd) 